\title{
Paranoia, sensitisation and social inference: findings from two large-scale, multi-round behavioural experiments
}

Barnby, J.M. ${ }^{1 *}$, Deeley, Q. ${ }^{2}$, Robinson, O. ${ }^{3}$, Raihani, N. ${ }^{4}$, Bell, V. ${ }^{1,5 \#}$, Mehta., M.A. ${ }^{1 \#}$

\section{Affiliations:}

${ }^{1}$ Social and Cultural Neuroscience Research Group, Centre for Neuroimaging Sciences, Institute of Psychiatry, Psychology, and Neuroscience, King's College London, London, UK

${ }^{2}$ Social and Cultural Neuroscience Research Group, Forensic and Neurodevelopmental Sciences, Institute of Psychiatry, Psychology, and Neuroscience, King's College London, London, UK

${ }^{3}$ Institute of Cognitive Neuroscience, University College London, London, UK.

${ }^{4}$ Psychology and Language Sciences, University College London, London, UK.

${ }^{5}$ Research Department of Clinical, Educational, and Healthy Psychology, University College London, London, UK.

${ }^{*}$ Corresponding Author

\#These authors contributed equally to the work

\section{Correspondence:}

Email: joe.barnby@kcl.ac.uk

Twitter: @joebarnby 


\section{Abstract:}

2 The sensitisation model suggests paranoia is explained by over-sensitivity to 3 perceived threat in social environments. However, this has been difficult to test 4 experimentally. We report two pre-registered studies that tested i) the sensitisation model as an explanation of paranoia, and; ii) the role of purported maintaining factors in supporting social sensitisation. In study one, we recruited a large general population sample $(N=987)$ who serially interacted with other participants in multiround Dictator games, matched to fair, partially fair, or unfair partners. Participants rated attributions of harmful intent and self-interest after each interaction. In study

10 two $(N=1011)$, a new sample of participants completed the same procedure and 11 additionally completed measures of anxiety, worry and interpersonal sensitivity. As predicted, paranoid ideation predicted higher and faster overall harmful intent attributions, whereas attributions of self-interest were unaffected, supporting the sensitisation model. Contrary to predictions, neither worry nor anxiety predicted harmful intent attributions while interpersonal sensitivity predicted decreased harmful intent attributions. In a third exploratory study we combined data sets to examine the effect of paranoia on trial by trial attributional changes when playing fair and unfair dictators. Paranoia predicted a greater reduction in harmful intent attributions when playing a fair but not unfair dictator, suggesting paranoia may also exaggerate the volatility of beliefs about the harmful intent of others. 


\subsection{Background}

23 Paranoia is a common feature in psychosis and involves an unfounded belief that others intend harm, now or in the future (1). Paranoid beliefs can be induced by recreational drugs $(2,3)$, following sleep deprivation (4) during or after seizures (5), or from being subject to high stress (6). Paranoia also exists as a continuous trait in the general population and has shown to be characterised by interpersonal sensitivity, mistrust, ideas of reference, and ideas of persecution $(7,8)$.

Once developed, paranoid beliefs are maintained by several personal and interpersonal factors. On the personal level, worry, insomnia (9) anxiety $(10,11)$, probabilistic reasoning biases (12), belief inflexibility (13), and safety behaviours (avoiding the source of perceived threat) (14) all contribute to paranoia. Interpersonal cognitive biases also affect how individuals interpret social situations. The most established effect is that those with paranoid beliefs have an externalising attribution bias, whereby causes of negative events are more likely to be attributed to other people (15). Trait interpersonal sensitivity has also been associated with paranoid thinking. Those at high risk of developing psychosis report increased paranoid thinking following simulated interactions in a virtual social environment which was predicted (16) or mediated (17) by interpersonal sensitivity.

The sensitisation model of psychosis argues that environmental stresses and genetic vulnerabilities sensitise biological, cognitive, and affective processes to produce symptoms of psychosis, and importantly, paranoid beliefs $(18,19,20)$. Neuroimaging studies have observed increased presynaptic dopamine leading up to (21) and during (22) the development of psychotic symptoms, suggesting aberrant dopaminergic transmission as crucial in sensitisation (23). Experimental data support the sensitisation of cognitive and affective processes that manifests as a 'jumping to conclusions' probabilistic reasoning bias $(12,24)$, high initial mistrust $(25,26)$ and more threatening or negatively valanced responses following heightened social arousal $(27,28)$.

One prediction arising from this model is that those high in paranoid ideation will show increased sensitivity to interpersonal interactions, and specifically potential or 
actual social threat, leading to an increased tendency to attribute harmful intent to others, putatively both more quickly and to a greater degree.

Economic games derived from game theory have been previously used to test the effect of paranoia on intention attributions. These games allow for live social interactions within a tightly controlled environment. Participants make decisions that have outcomes with genuine gains and losses and therefore real, albeit small, harms and benefits $(29,25)$. Existing research has shown that increases in harmful intent attributions are associated with trait paranoia, social threat $(29,30)$, social cohesion of task partners (31), and increased relative social rank, and outgroup status, of the interaction partner (32). However, current game theory paradigms in paranoia research that have allowed for participant-to-participant (rather than simulated; 16, 17 ) interactions have tended to use single round games or brief interactions that are not able to test the effect of paranoia and additional psychological variables on attributions over evolving interactions.

In this study, we implemented a multiple-round game theory interaction using serial Dictator games. The Dictator game has been used widely in paranoia research (29, 30,32 ) and involves a situation where two participants are paired and one (the 'dictator') is given a sum of money that they can choose to share with the 'receiver' participant (33). The receiver has no control and must accept any amount that the dictator offers. After each interaction, receivers are required to rate to what extent the dictators were motivated by self-interest or an intent to harm. In the paradigm developed for this study, participants completed six serial Dictator trials against fair, partially fair and unfair partners, while rating harmful intent and self-interest motivating their partner's actions, allowing a test of sensitivity over evolved social interactions. This also allowed us to test the effect of several key affective processes previously identified as important in paranoia, namely anxiety, worry, and interpersonal sensitivity.

The sensitisation model of paranoia suggests several hypotheses we tested over two studies. In study one, we hypothesised that high levels of paranoid ideation would predict earlier and larger harmful intent attributions during the multi-round interaction. In study two we hypothesised that harmful intent attributions would be predicted by anxiety, interpersonal sensitivity, and worry. Studies one and two were pre- 
registered and included hypotheses designed to replicate findings from previous studies (high attribution of harmful intent is associated with higher paranoia and unfair dictators; 29-32) as well as the key experimental hypotheses described above. In study three we combined data from study one and two to complete exploratory analysis to gain better resolution on trial by trial effects, dictator exposure effects, and dictator behaviour overall as each independent sample from study one and two gave varying results.

\subsection{Study 1}

This study tested the main hypothesis that paranoid ideation predicts the in-themoment harmful intent attributions within serial interpersonal interactions, both in terms of overall value and by how quickly individuals reach a marker of high harmful intent attribution. The full list of pre-registered hypotheses is given below.

\subsection{Methodology}

This project was approved by the Kings College London ethics board (Study 1: MRS-17/18-8312). All data were collected in September 2018 using Prolific Academic (hereafter Prolific; www.prolific.ac), an online crowdsourcing platform. All data and analysis scripts are available online (https://osf.io/u92rg/).

Prior to taking part in both studies, participants were informed that their participation was voluntary, and were required to tick a box giving consent for the authors to use their anonymous data for research purposes. Using Prolific allowed rapid recruitment of a more demographically diverse sample of participants than recruitment from our social media or university networks (34). We included participants from the UK who were fluent in English and had no current or history of mental illness.

We recruited 987 participants (372 males). Participants first completed the Green Paranoid Thoughts Scale (GPTS; 35). Participants were asked to indicate the extent of feelings described in 32 statements using a Likert Scale of 1 to 5 , where $1=$ Not at All and $5=$ Totally. Scores can range from $32-160$, with higher scores indicating a greater degree of paranoia. The GPTS was chosen as a suitable 
113 measure as it includes both core aspects of the definition of paranoia (1): social

114 concerns about others and perception of intended harm. It has also shown to be

115 the most reliable and valid scale for measuring paranoia across the clinical and

116 non-clinical spectrum (36). Total paranoia scores were obtained for each

117 participant by summing the response scores to all questions, comprising both the

118 social reference and the persecution scales. Hereafter, this variable is referred to 119 as 'paranoia'.

120 After completing the survey, and in keeping with Raihani and Bell $(29,30)$ we

121 allowed a minimum interval of 7 days to elapse before inviting participants to take

122 part in the multi-round dictator game.

123 We developed a within-subjects, multi-trial modification of the Dictator game 124 design used in previous studies to assess paranoia (See Appendix A; 29, 30).

125 Each participant played six trials against three different types of dictator. In each

126 trial, participants were told that they had been endowed with a total of $£ 0.10$ and

127 their partner (the dictator) had the choice to take half ( $£ 0.05)$ or all $(£ 0.10)$ the

128 money from the participant. Dictators were set to either always take half of the

129 money, have a 50:50 chance to take half or all of the money, or always take all of

130 the money. This was noted in this study as Fair, Partially Fair, and Unfair,

131 respectively. The order that participants were matched with dictators was

132 randomised. Each dictator had a corresponding cartoon avatar with a neutral

133 expression to support the perception that each of the six trials was with the same 134 partner.

135 After each trial, participants were asked to rate on a scale of 1-100 (initialised at

136 50) to what degree they believed that the dictator was motivated a) by a desire to

137 earn more (self-Interest) and b) by a desire to reduce their bonus in the trial

138 (harmful intent). Following each block of six trials, participants were asked to rate

139 the character of the dictator overall by scoring intention again on both scales.

140 Therefore, participants judged their perceived intention of the dictator on both a

141 trial-by-trial and summary level.

142 After making all 42 attributions (two attributions for each of the 6 trials over 3

143 partners, plus three additional overall attributions for each partner), participants were 
144 put in the role of the dictator for 6 trials - whether to make a fair or unfair split of

$145 £ 0.10$. Participants were first asked to choose an avatar from nine different cartoon

146 faces before deciding on their 6 different splits. These dictator decisions were not

147 used for analysis but were collected to truthfully inform participants that decisions

148 were made by real people.

149 This modification to the original dictator game design allowed us to track how 150 changes in pre-existing paranoia where associated with changes in attributions 151 about partner behaviour and the order of initial partner exposure, and whether 152 attributions were highly variables over trials or consistent. We recruited 812 153 participants (294 males) back to play the multi-round dictator game. The mean age 154 range of participants was $36-40$ in the second sample.

155 All participants were paid for their completion of the GPTS, regardless of follow up.

156 Participants were paid a baseline payment for their completion of the dictator 157 game, along with any additional bonuses won in the game.

Preregistered predictions (https://aspredicted.org/ka4ny.pdf)

1. High trait paranoia (as measured by GPTS) will be associated with increased attribution of harmful intent to partners across all trials (but trait paranoia will not be associated with variation in attributions of self-interest).

2. Attribution of harmful intent to different dictators will follow a dose-response relationship (fair < partially fair < unfair) across all ranges of paranoia. However, those will high paranoia will have a higher baseline of average harmful intent. There will be no interaction between trait paranoia and dictator fairness on attribution of harmful intent.

3. High trait paranoia will be associated with reaching a peak in harmful intent attribution (defined as a score of 60 or more) in fewer trials when analysing each participant but no difference in attribution of self-interest within each dictator.

171 All analyses conform to those outlined in our preregistration unless stated otherwise. 
172 This study used an information-theoretic approach for all confirmatory analysis. We

173 analysed the data using multi-model selection with model averaging (described in

174 29, 30). The Akaike information criterion, corrected for small sample sizes (AICc),

175 was used to evaluate models, with lower AICc values indicating a better fit (37). The

176 best models are those with the lowest AICc value. To adjust for the intrinsic

177 uncertainty over which model is the true 'best' model, we averaged over the models

178 in the top model set to generate model-averaged effect sizes and confidence

179 intervals (38). In addition, parameter estimates, and confidence intervals are

180 provided with the full global model to robustly report a variable's effect in a model

181 (39). This used package "MuMIn" (40). All analyses were conducted in R (41). All

182 visualisations were generated using the package 'ggplot2' (42).

183 In our models, all baseline continuous scale scores were centred and scaled to 184 produce $Z$ values. All model statistics reported are beta coefficients.

Average scores of harmful intention attributions and self-interest for each dictator were taken over each six trials for trial analysis. These were used for cumulative link mixed-models (clmm; 43). Harmful intent and self-interest attributions were set as our dependent variable. Paranoia, dictator order, dictator behaviour (fair, unfair,

189 partially fair), age, sex, and paranoia $\mathrm{x}$ dictator behaviour were set as our

190 explanatory terms with ID set as the random term.

191 For our third prediction, participants that scored above 60 were considered to have scored high harmful intent attributions. Both harmful intent and self-interest scores participants were set a value of 6 if they had scored 60 in their first trial, 5 if they had scored over 60 by their second trial, 4 if they had scored 60 by their third trial, and so on. We report this result, but also wanted to consider a high harm attribution as someone that scored over the mean harmful intent attribution of the population for each dictator. This is also reported in addition to our preregistered plan, which was based on previous mean group estimates. Mean thresholds for each dictator are stated for each analysis in the Results. All trials following the threshold being reached were coded as 0 . Participants not reaching the threshold for any trial were coded 0 across all trials. Both unfair and fair dictator behaviour were analysed with two cumulative link models ( $\mathrm{clm}$ ) each, one for harm-intent and one for self-interest.

203 This slightly deviates from our preregistration that suggests the use of Kruskal-Wallis 
204 and Dunn post-hoc tests, however we decided that using a clm is a more robust way

205 to analyse the data.

206 For visualisation purposes we calculated paranoia groups based on the quantiles of 207 GPTS scores across the population, and additionally divided those in the top quantile 208 by those exceeding the clinical mean of paranoia defined in previous work (101.9; 209 35). These divisions were: Low (<36; $n=232$ ) Medium (36-43; $n=180)$, High (44210 59; $n=199)$, and Very High (59-101.9; $n=167)$, and Clinical $(>102, n=34)$. This 211 variable is hereafter named paranoia 'level'. Slightly different score parameters for

212 each paranoia level were included in our pre-registration but we have adapted them 213 in this study based on our population GPTS quartiles. 


\subsection{Results}

216812 participants were included in the analysis. 15 were removed for incomplete 217 data, 24 removed for failing both control questions, and 136 for non-participation

218 in the multi-round dictator game. Mean baseline paranoid ideation in the excluded 219 participants $(M=50.43$, $S E=1.62$, range $=32-134)$ were comparable to those 220 that were included in the analysis $(\mathrm{t}(252)=0.322,95 \% \mathrm{Cl}:-2.93,4.08)$.

221 Explanatory variables of baseline paranoia score

222 Paranoia scores ranged from 32-149 with a mean of 51 (SE: 0.74; Skew: 1.7). Older 223 participants were less paranoid $(-1.89 ; 95 \% \mathrm{Cl}:-2.22,-1.57)$, male participants were 224 more paranoid $(0.17 ; 95 \% \mathrm{Cl}: 0.04,0.34)$, and there was no effect of education on 225 paranoia $(-0.39 ; 95 \% \mathrm{Cl}:-1.16,0.17)$.

226 Prediction 1: Paranoia and harmful intent

227 As predicted, paranoia positively predicted higher $\mathrm{HI}$ attributions across all three 228 dictators $(0.36,95 \% \mathrm{Cl}: 0.19,0.53$; Table 1$)$. There was no effect of paranoia on SI 229 attributions $(0.01,95 \% \mathrm{Cl}:-0.09,0.11)$.

230 Prediction 2: Dictator behaviour and harmful intent

231 As predicted, as dictators were increasingly unfair (higher proportion of unfair 232 decisions), higher $\mathrm{HI}$ attributions were observed (Table 1). SI attributions also 233 increased as a result of unfair dictator behaviour in a similar manner.

234 Figure 1 depicts the difference in $\mathrm{HI}$ and $\mathrm{SI}$ attributions between the population when 235 delineated by their paranoia level (low, medium, high, very high, clinical) for both 236 Study 1 and Study 2.

\section{Prediction 3: Paranoia and earlier high harmful intent attributions}

As predicted, high (over 60) harmful intent attributions were triggered in earlier trials as paranoia increases for both unfair $(-0.12 ; 95 \% \mathrm{Cl}:-0.21,-0.03)$ and fair $(-0.14$, 95\% Cl: $-0.33,-0.01$ ) dictators. This was not found for SI attributions (see Appendix B). 


\section{Exploratory analysis}

244 We also completed an analysis using a relative threshold for earlier high decisions

245 based on the mean of the population for each dictator rather than a pre-set cut-off of

24660 as in the preregistered analysis. For unfair dictators, high (mean $=53.51) \mathrm{HI}$

247 attributions were triggered in earlier trials as paranoia increased $(-0.12 ; 95 \% \mathrm{Cl}$ :

$2480.20,-0.02)$. However, this wasn't found for fair dictators (mean = 24.26) $(-0.06 ; 95 \%$

$249 \mathrm{Cl}:-0.19,0.01)$. This was not found for SI attributions in either dictator condition.

250 See figure 2 (a) for trial-by-trial average attributions across participants for study 1 251 and 2 . 
Table 1. Variables effecting Harmful Intention and Self Interest scores in the multi-round dictator game (Study 1). Harmful Intent was coded as a five-level ordinal categorical variable and set as the response term in the clmm. ID was set as the random variable (43). Relative Importance is the probability that the term in question is a component of the true best model and a value for the amount of times the term is included in the selection of top models to be averaged. Order refers to the order in which a fair, partially fair, or unfair dictator was presented to participants.

\begin{tabular}{|c|c|c|c|c|c|}
\hline \multirow[t]{2}{*}{ Parameter } & \multirow[t]{2}{*}{ Estimate } & \multirow{2}{*}{$\begin{array}{l}\text { Standard } \\
\text { Error }\end{array}$} & \multicolumn{2}{|c|}{$95 \% \mathrm{Cl}$} & \multirow{2}{*}{$\begin{array}{c}\text { Relative } \\
\text { Importance }\end{array}$} \\
\hline & & & Lower & Upper & \\
\hline \multicolumn{6}{|l|}{ Harmful Intent Attributions } \\
\hline Intercept 1|2 & -1.26 & 0.11 & -1.48 & -1.05 & \\
\hline Intercept 2|3 & 0.47 & 0.10 & 0.27 & 0.68 & \\
\hline Intercept 3|4 & 2.17 & 0.12 & 1.94 & 2.39 & \\
\hline Intercept $4 \mid 5$ & 3.67 & 0.14 & 3.41 & 3.94 & \\
\hline $\begin{array}{l}\text { Dictator } \\
\text { (Fair < Partially Fair < Unfair) }\end{array}$ & 2.22 & 0.09 & 2.06 & 2.39 & 1 \\
\hline $\begin{array}{l}\text { Order } \\
\text { (Fair < Partially Fair < Unfair) }\end{array}$ & -1.12 & 0.15 & -1.42 & -0.83 & 1 \\
\hline Paranoia (Z score) & 0.36 & 0.09 & 0.19 & 0.53 & 1 \\
\hline Dictator x Paranoia & 0.14 & 0.10 & -0.06 & 0.34 & 0.79 \\
\hline Sex (Male) & -0.03 & 0.11 & -0.26 & 0.19 & 0.25 \\
\hline \multicolumn{6}{|l|}{ Self Interest Attributions } \\
\hline Intercept $1 \mid 2$ & -6.53 & 0.25 & -7.01 & -6.05 & \\
\hline Intercept 2|3 & -5.25 & 0.21 & -5.66 & -4.84 & \\
\hline Intercept 3|4 & -3.15 & 0.16 & -3.46 & -2.84 & \\
\hline Intercept $4 \mid 5$ & -0.28 & 0.11 & -0.50 & -0.07 & \\
\hline $\begin{array}{l}\text { Dictator } \\
\text { (Fair < Partially Fair < Unfair) }\end{array}$ & 4.33 & 0.17 & 3.99 & 4.67 & 1 \\
\hline $\begin{array}{l}\text { Order } \\
\text { (Fair < Partially Fair < Unfair) }\end{array}$ & -0.82 & 0.16 & -1.13 & -0.50 & 1 \\
\hline Paranoia (Z score) & 0.01 & 0.05 & -0.09 & 0.11 & 0.24 \\
\hline Sex (Male | Female) & -0.03 & 0.11 & -0.23 & 0.18 & 0.23 \\
\hline
\end{tabular}




\subsection{Study 2}

263 We tested whether interpersonal sensitivity (Interpersonal Sensitivity Measure; ISM)

264 (44), state and trait anxiety (STAI) (45) and worry (46) - key affective processes

265 involved in paranoid ideation - would account for within-group harmful intent

266 attributions. The full list of pre-registered hypotheses is given below.

\section{$267 \quad 3.1$ Methodology}

268 This project was approved by the Kings College London ethics board (Study 2: LRS18/19-9281). Data were collected in February 2019 using Prolific. All data and analysis scripts are available online (https://osf.io/u92rg/).

271 We recruited 1011 participants (374 males). Participants recruited for this study were

272 not participants in Study 1. All study procedures and analyses were identical to

273 Study 1 aside from the inclusion of anxiety, worry and interpersonal sensitivity

274 measures.

275 We assessed both trait anxiety and state anxiety using the STAI (45). It is comprised

276 of two subscales, one for trait and one for state anxiety, each made of 20 items.

277 Each item is rated on a scale of one to four, from "Almost Never" to "Almost Always".

278 The trait measure was given to participants at baseline alongside the GPTS. The

279 state measure was given immediately after the multi-round dictator game.

280 We measured interpersonal sensitivity using the ISM (44). The ISM is comprised of

281 five subscales: Fragile Inner Self (5 items), Need for Attachment (8 items),

282 Interpersonal Awareness (7 items), Timidity (8 items), and Separation Anxiety (8

283 items). Each item is on a scale of one to four, from "Very Unlike You" to "Very Like

284 You". Subscales are summed to form summary scores. The ISM was given at 285 baseline alongside the GPTS.

286 We also measured worry using the Penn-State Worry Questionnaire (PSWQ) (46) as

287 worry has been additionally implicated as highly predictive of paranoia (1). The

288 PSWQ is comprised of 16 items, each on a scale of one to five, from "Not at all

289 typical of me" to "Very typical of me". The PSWQ was given at baseline alongside 290 the GPTS. 
291 All analyses conform to our preregistration unless stated otherwise.

292

Preregistered predictions (http://aspredicted.org/yz5gr.pdf)

293 We pre-registered the following predictions:

294 1. State anxiety will be associated with increased harmful intent attributions (but not

295 self-interest attributions) to partners when averaged across all trials within each

296 partner.

297 2. There will be an interaction between state anxiety and trait paranoia leading to

298 increased attribution of harmful intent (but not self-interest attributions) to

299 partners across all trials.

3003 3. High interpersonal sensitivity (as defined by Boyce \& Parker) scores will be associated with increased harmful intent attributions (but not self-interest attributions) to partners when averaged across all trials within each partner.

4. High scores on interpersonal sensitivity subscales of 'Fragile Inner Self' and 'Interpersonal Awareness' will be associated with increased harmful intent attributions (but not self-interest attributions) to partners when averaged across all trials within each partner.

5. There will be an interaction between state anxiety and trait paranoia leading to a decreased number of trials before a high ( $>$ mean) attribution score of harmful intent (but not self-interest attributions) to partners is triggered separately across unfair and fair dictators.

311 We included the explanatory variables from the STAI, PSWQ and ISM in our

312 cumulative link mixed models alongside the GPTS scores with ID set as the random

313 variable. All continuous variables were z-score transformed. All model statistics

314 reported are beta coefficients unless stated otherwise. 


\subsection{Results}

317885 participants remained in the analysis. 8 were removed for incomplete data and

318118 for non-participation in the multi-round dictator game. Mean baseline paranoid

319 ideation in the excluded participants $(M=58.54, S E=2.35$, range $=32-140)$ were

320 higher than participants that were included in the analysis $(\mathrm{t}(153)=-2.41,95 \% \mathrm{Cl}$ :

$321-10.85,-1.09)$ by a small amount.

\section{Explanatory variables of baseline paranoia}

323 Paranoia scores ranged from 32-159 with a mean of 53 (SE: 0.45; Skew: 1.54).

324 Older participants were less paranoid $(-0.05 ; 95 \% \mathrm{Cl}:-0.05,-0.04)$, there was a

325 negligible effect of being male on paranoia $(0.05 ; 95 \% \mathrm{Cl}:-0.04,0.24)$, and there

326 was a quadradic $(-1.20,95 \% \mathrm{Cl}:-1.80,-0.60)$ relationship between education and

327 paranoia. Paranoia positively correlated with all baseline variables (see Figure 3).

328 For a detailed distribution of the data see Appendix C.

329 Replication of main findings of study 1

330 Paranoia positively predicted higher $\mathrm{HI}$ attributions across all three dictators. There

331 was no effect of paranoia on SI attributions. Additionally, unfairness of dictator was

332 associated with higher $\mathrm{HI}$ and $\mathrm{SI}$ attributions. Order effects were also replicated. See

333 Figure 1 and Table 2.

334 For unfair dictators, high (mean $=46.56) \mathrm{HI}$ attributions were not uniformly observed

335 in earlier trials as paranoia increased $(-0.06 ; 95 \% \mathrm{Cl}:-0.17,0.01)$, but were for fair

336 dictators $($ mean $=21.39)(-0.12 ; 95 \% \mathrm{Cl}:-0.20,-0.03)$. Paranoia was not associated

337 with high SI attributions in earlier trials in either dictator condition.

338 Figure $2 \mathrm{~b}$ shows average trial by trial attributions for each level of paranoia in Study

3392. 
Table 2. Variables effecting Harmful Intent and Self Interest scores in the multiround dictator game. Harmful Intent was coded as a five-level ordinal categorical variable and set as the response term in the clmm. ID was set as the random variable (43). Relative Importance is the probability that the term in question is a component of the true best model and a value for the amount of times the term is included in the selection of top models to be averaged. Order refers to the order in which a fair, partially fair, or unfair dictator was presented to participants.

\begin{tabular}{|c|c|c|c|c|c|}
\hline \multirow[t]{2}{*}{ Parameter } & \multirow[t]{2}{*}{ Estimate } & \multirow{2}{*}{$\begin{array}{l}\text { Standard } \\
\text { Error }\end{array}$} & \multicolumn{2}{|c|}{$95 \% \mathrm{Cl}$} & \multirow{2}{*}{$\begin{array}{c}\text { Relative } \\
\text { Importance }\end{array}$} \\
\hline & & & Lower & Upper & \\
\hline \multicolumn{6}{|l|}{ Harmful Intent Attributions } \\
\hline Intercept 1|2 & -0.64 & 0.23 & -1.09 & -0.18 & \\
\hline Intercept 2|3 & 1.28 & 0.24 & 0.82 & 1.74 & \\
\hline Intercept 3|4 & 2.95 & 0.25 & 2.47 & 3.43 & \\
\hline Intercept $4 \mid 5$ & 4.38 & 0.26 & 3.88 & 4.89 & \\
\hline $\begin{array}{l}\text { Dictator } \\
\text { (Fair < Partially Fair < Unfair) }\end{array}$ & 2.00 & 0.09 & 1.82 & 2.18 & 1 \\
\hline $\begin{array}{l}\text { Order } \\
\text { (Fair < Partially Fair < Unfair) }\end{array}$ & -1.17 & 0.17 & -1.52 & -0.83 & 1 \\
\hline Paranoia (Z score) & 0.35 & 0.10 & 0.15 & 0.54 & 1 \\
\hline Sex (Male | Female) & -0.16 & 0.21 & -0.71 & 0.10 & 0.52 \\
\hline Age & 0.00 & 0.01 & -0.01 & 0.02 & 0.32 \\
\hline \multicolumn{6}{|l|}{ Self Interest Attributions } \\
\hline Intercept 1|2 & -6.59 & 0.35 & -7.27 & -5.91 & \\
\hline Intercept 2|3 & -5.35 & 0.33 & -5.99 & -4.71 & \\
\hline Intercept 3|4 & -3.16 & 0.30 & -3.75 & -2.58 & \\
\hline Intercept $4 \mid 5$ & -0.21 & 0.28 & -0.75 & 0.33 & \\
\hline $\begin{array}{l}\text { Dictator } \\
\text { (Fair < Partially Fair < Unfair) }\end{array}$ & 4.59 & 0.17 & 4.26 & 4.93 & 1 \\
\hline $\begin{array}{l}\text { Order } \\
\text { (Fair < Partially Fair < Unfair) }\end{array}$ & -0.71 & 0.16 & -1.02 & -0.39 & 1 \\
\hline Age & 0.00 & 0.01 & -0.02 & 0.00 & 0.43 \\
\hline Paranoia (Z score) & -0.03 & 0.07 & -0.28 & 0.09 & 0.34 \\
\hline Sex (Male | Female) & 0.01 & 0.07 & -0.31 & 0.43 & 0.11 \\
\hline
\end{tabular}


A

$$
n=812
$$

Fair

100

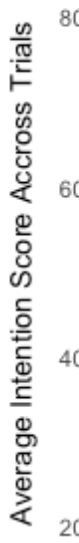

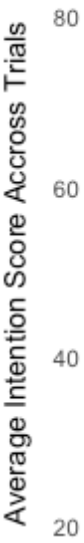
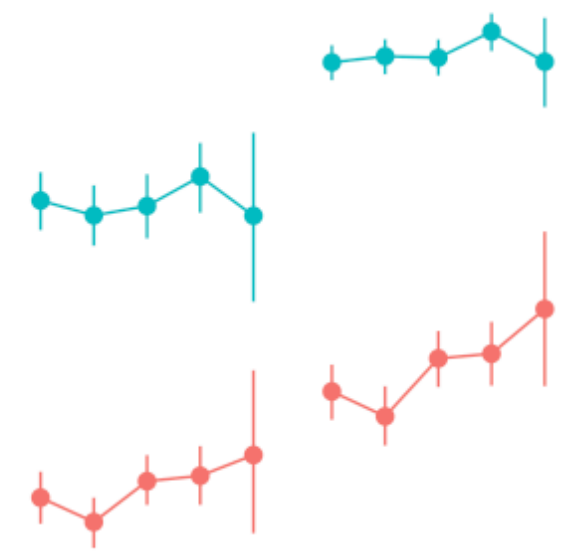

B

$$
n=885
$$

Fair

Partially Fair

Unfair

100
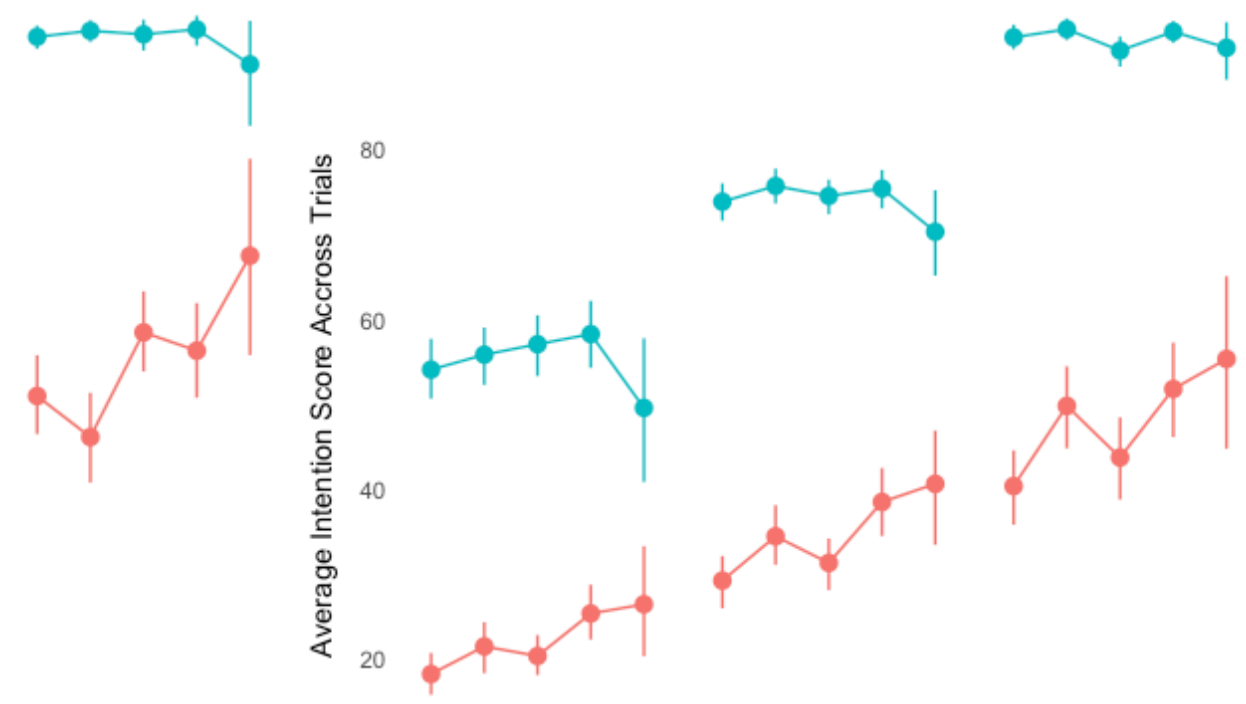

0
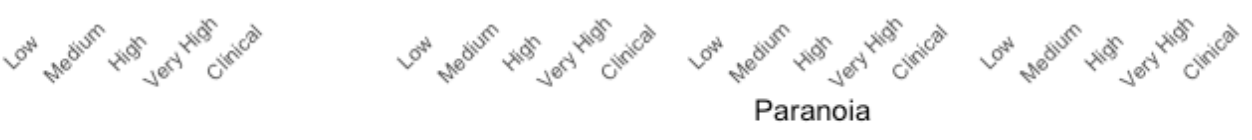

type $-\phi$ Harm Intention $-\phi-$ Self Interest

351 Figure 1. Average Self-Interest attributions (Blue) and Harmful Intent attributions (Red), averaged across trials for divisions of GPTS score and faceted by each type of dictator for both study 1 (A) and study 2 (B). Dots represent the mean for each level of paranoia. Lines represent the $95 \%$ confidence interval. Participants played against different partners in a pseudo-random order. 'Clinical' refers to participants in the general population who scored past the threshold for GPTS scores typical in clinical populations $(101.9 ; 35)$. We found that

358 the degree of fairness proportionally scaled harmful intent scores across all levels of

359 paranoia. This was also true for self-interest scores. However, paranoia increased

360 harmful intent scores within each condition. This was not true for self-interest scores. 

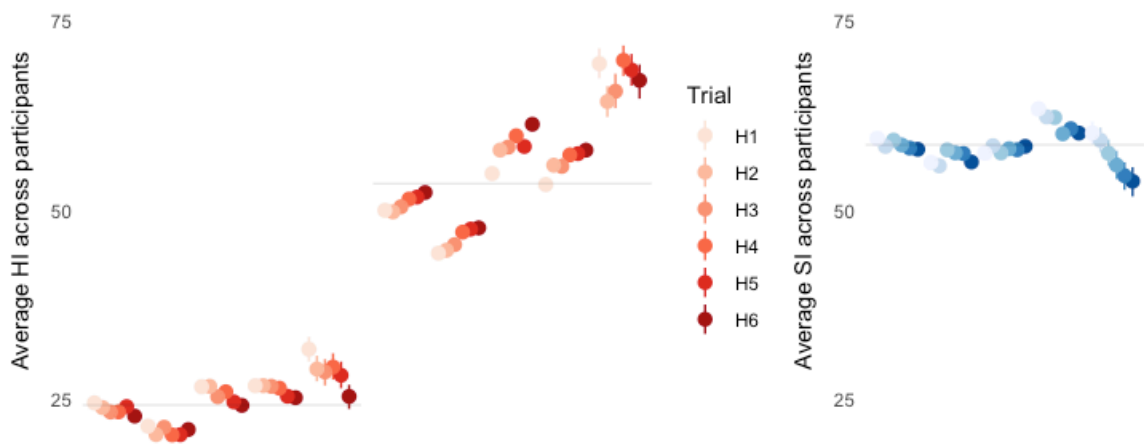

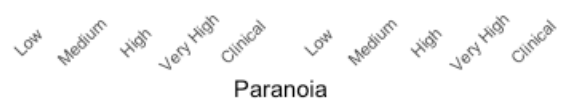

Fair

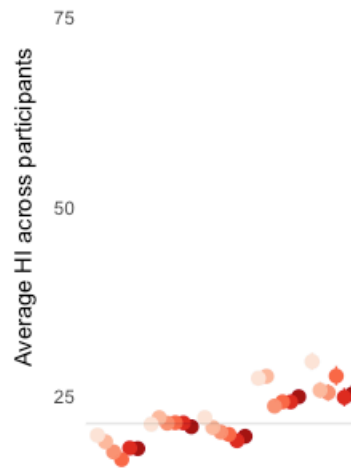

Paranoia

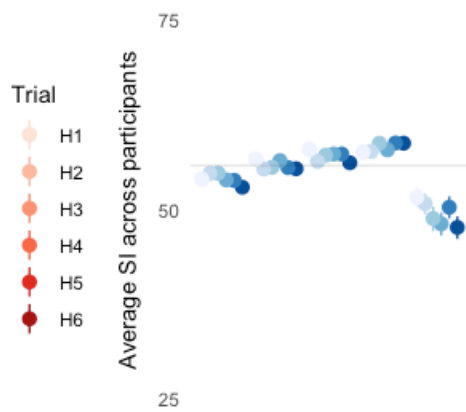

Trial

Figure 2. Average Harmful Intent (Red) and Average Self-Interest (Blue) attributions for each trial across divisions of GPTS scores, faceted by type of dictator for study 1 (A) and study 2 (B). Points = mean, bars $=95 \%$ confidence interval. Grey lines $=$ mean score across the group. 'Clinical' refers to participants in the general population who scored past the threshold for GPTS scores typical in clinical populations $(101.9 ; 35)$. 
$370 \quad$ Predictions 1 and 2: State anxiety, paranoia and harmful intent

371 Contrary to predictions, state anxiety did not predict overall $\mathrm{HI}$ or SI attributions in

372 any dictator condition and there was no interaction with paranoia.

373 Prediction 3 and 4: Interpersonal sensitivity, paranoia and harmful intent

374 Contrary to predictions, interpersonal sensitivity predicted a decrease in overall HI

375 but not SI attributions across all dictators. There was no interaction between

376 interpersonal sensitivity and paranoia for $\mathrm{HI}$ or SI attributions across all dictators.

377 Similarly, the "interpersonal awareness" subscale of the ISM was associated with a

378 decrease in $\mathrm{HI}$ attributions overall across dictators and the 'separation anxiety'

379 subscale of the ISM also was associated with an increase in $\mathrm{HI}$ attributions overall

380 across dictators. The 'need for attachment' and 'timidity' subscales were not

381 associated with an increase in HI attributions. The 'Interpersonal awareness'

382 subscale score of the ISM was associated with increased SI attributions overall

383 across dictators. 'Need for attachment' subscale scores of the ISM was associated

384 with increased SI attributions and 'timidity' predicted decreased SI attributions overall

385 across dictators.

386 Paranoia remained a strong predictor of $\mathrm{HI}$ attributions but not SI attributions for all

387 models. See Table 3 for the effect sizes for all predictors.

388 Prediction 5: Anxiety, paranoia, and trials to peak decision

389 Contrary to predictions, state anxiety alone and its interaction with paranoia didn't

390 predict scoring above the mean in an earlier trial for $\mathrm{HI}$ and $\mathrm{SI}$ attributions during

391 both unfair and fair dictators. 
Table 3. Summary of extra explanatory variables effecting Harmful Intention and Self Interest attributions in the multi-round dictator game (Study 2). Harmful Intent was coded as a five-level ordinal categorical variable and set as the response term in the clmm. ID was set as the random variable (42). Relative Importance is the probability that the term in question is a component of the true best model. Numbers denote the model that the parameter belonged to for each outcome variable. E.g. under Harmful intent Attributions, Separation Anxiety and Timidity were run in the same model (4) but separate to Trait Anxiety (1). NA = not included in the final top model. Paranoia was included as an independent variable in each model.

\begin{tabular}{|c|c|c|c|c|c|c|}
\hline \multirow[t]{2}{*}{ Model } & \multirow[t]{2}{*}{ Parameter } & \multirow[t]{2}{*}{ Estimate } & \multirow{2}{*}{$\begin{array}{l}\text { Standard } \\
\text { Error }\end{array}$} & \multicolumn{2}{|c|}{$95 \% \mathrm{Cl}$} & \multirow{2}{*}{$\begin{array}{c}\text { Relative } \\
\text { Importance }\end{array}$} \\
\hline & & & & Lower & Upper & \\
\hline & \multicolumn{6}{|l|}{ Harmful Intent Attributions } \\
\hline 1 & Trait Anxiety & -0.19 & 0.12 & -0.42 & 0.05 & 1 \\
\hline 2 & State Anxiety & 0.00 & 0.04 & -0.19 & 0.23 & 0.14 \\
\hline 3 & Interpersonal Sensitivity & -0.29 & 0.12 & -0.52 & -0.06 & 1 \\
\hline 4 & Interpersonal Awareness & -0.54 & 0.13 & -0.80 & -0.28 & 1 \\
\hline 4 & Separation Anxiety & 0.36 & 0.14 & 0.08 & 0.64 & 1 \\
\hline 4 & Timidity & -0.02 & 0.07 & -0.34 & 0.14 & 0.22 \\
\hline 4 & Need for Attachment & -0.01 & 0.03 & -0.09 & 0.07 & 0.19 \\
\hline 4 & Fragile Inner Self & 0.01 & 0.07 & -0.22 & 0.38 & 0.18 \\
\hline 5 & Worry & -0.06 & 0.06 & -0.18 & 0.05 & 1 \\
\hline \multirow[t]{2}{*}{$1-5$} & Paranoia (Range) & $\begin{array}{c}0.34- \\
0.60\end{array}$ & $\begin{array}{c}0.06- \\
0.14\end{array}$ & $\begin{array}{c}0.13- \\
0.38 \\
\end{array}$ & $\begin{array}{c}0.54- \\
0.88 \\
\end{array}$ & $1-1$ \\
\hline & \multicolumn{6}{|l|}{ Self Interest Attributions } \\
\hline 6 & Trait Anxiety & 0.05 & 0.08 & -0.06 & 0.29 & 0.41 \\
\hline 7 & State Anxiety & 0.14 & 0.12 & -0.01 & 0.38 & 0.76 \\
\hline 8 & Interpersonal Sensitivity & NA & NA & NA & NA & NA \\
\hline 9 & Interpersonal Awareness & 0.31 & 0.14 & 0.04 & 0.58 & 1 \\
\hline 9 & Separation Anxiety & -0.02 & 0.07 & -0.38 & 0.15 & 0.16 \\
\hline 9 & Timidity & -0.46 & 0.11 & -0.68 & -0.23 & 1 \\
\hline 9 & Need for Attachment & 0.28 & 0.11 & 0.07 & 0.48 & 1 \\
\hline 9 & Fragile Inner Self & -0.05 & 0.10 & -0.38 & 0.10 & 0.35 \\
\hline 10 & Worry & 0.18 & 0.12 & 0.01 & 0.40 & 0.86 \\
\hline $6-10$ & Paranoia (Range) & $\begin{array}{c}-0.03- \\
-0.10\end{array}$ & $\begin{array}{c}0.05- \\
0.11 \\
\end{array}$ & $\begin{array}{c}-0.28- \\
-0.35\end{array}$ & $\begin{array}{c}0.02- \\
0.11 \\
\end{array}$ & $\begin{array}{c}0.31- \\
0.58\end{array}$ \\
\hline
\end{tabular}




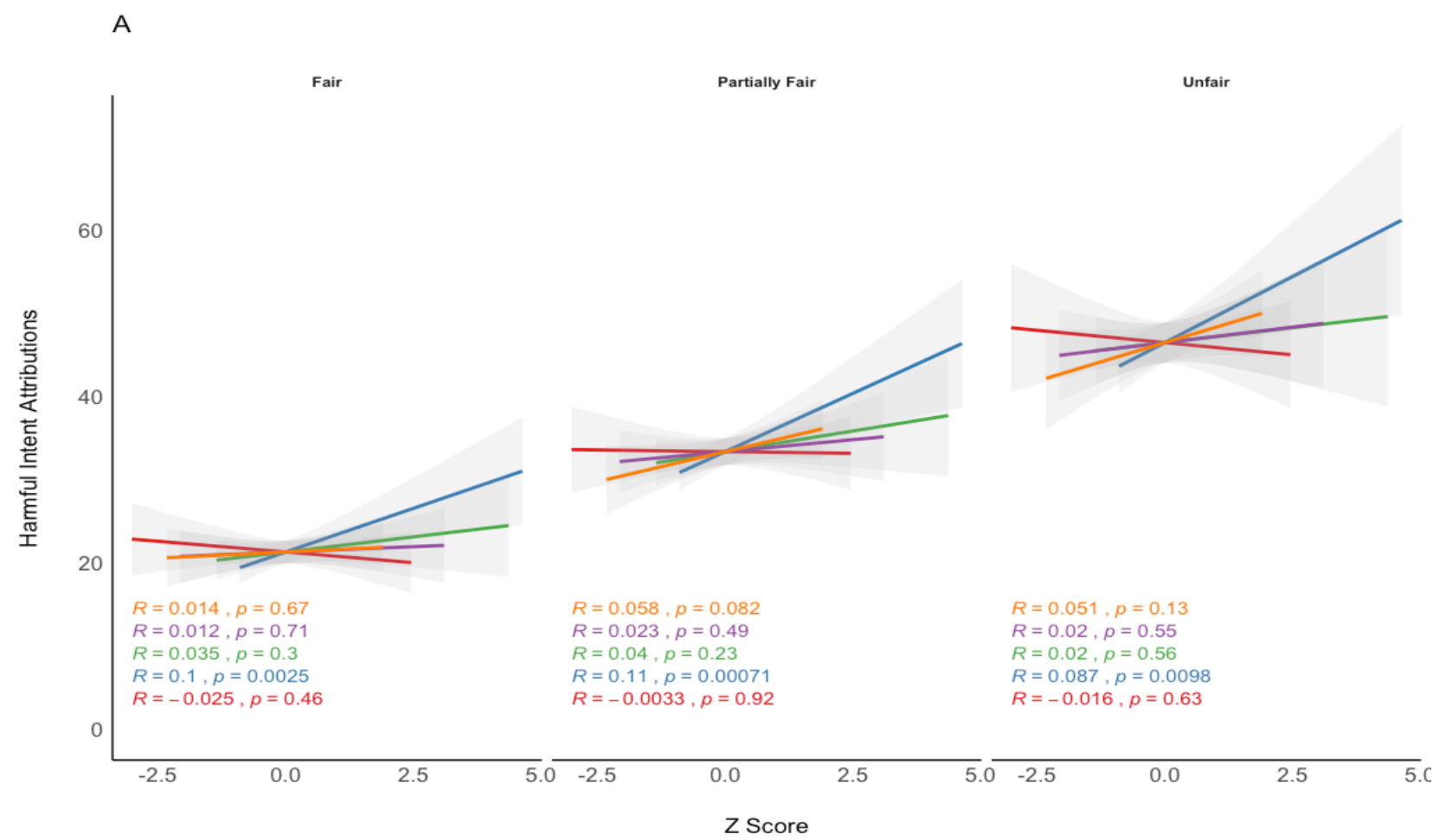

B

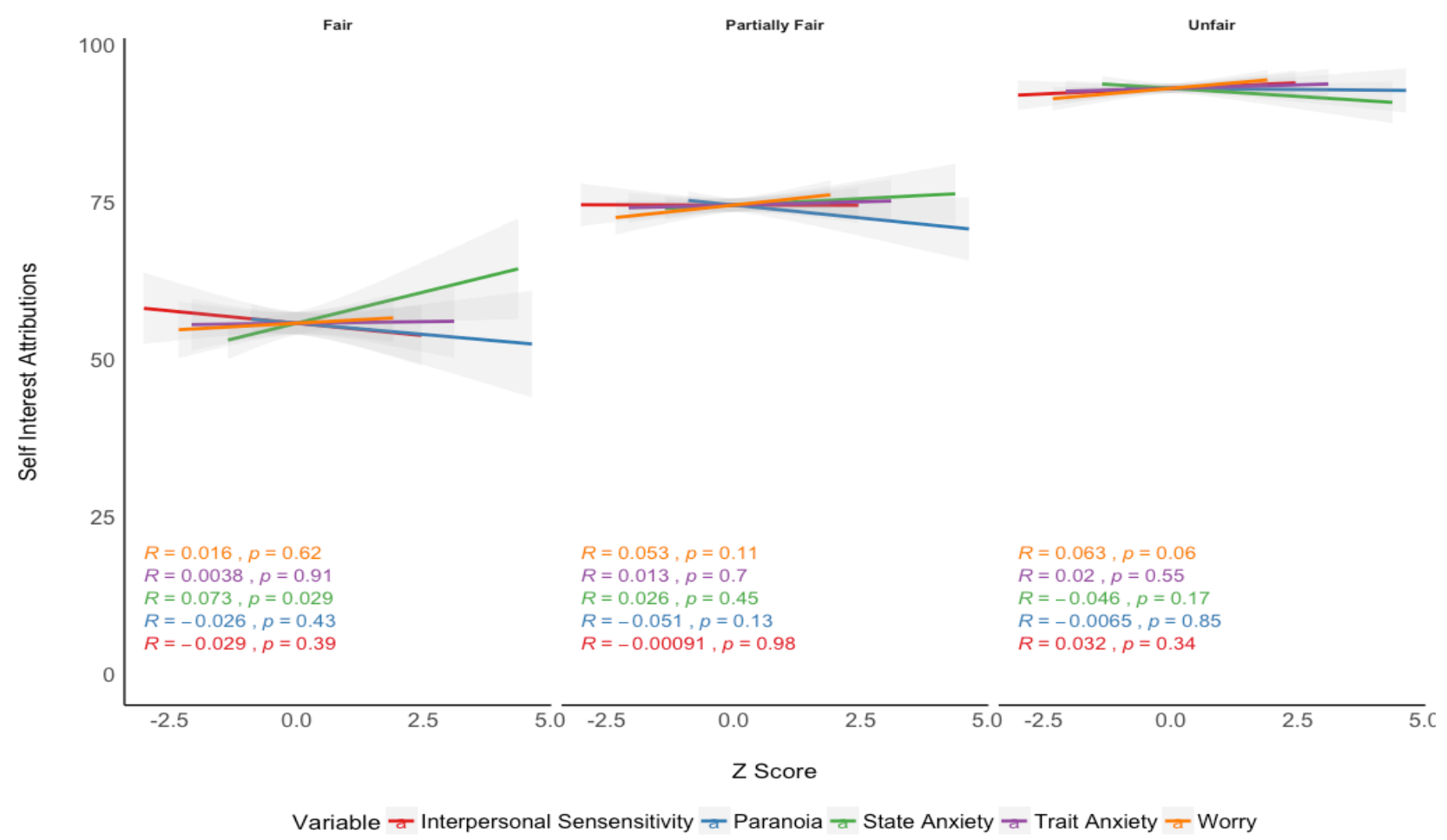

406

407 Figure 3. Pearson R correlations for centred and scaled scores on state and 408 trait anxiety, paranoia, interpersonal sensitivity, and worry questionnaires by 409 harmful intent (A) and self-interest (B) scores in Study 2, faceted by dictator 410 condition. Paranoia is the only significant measure correlated with harmful intent 411 attributions. It is not correlated with self-interest attributions. $N=885$. 


\section{$412 \quad 4.0$ Study 3}

413 We combined data from Study 1 and 2 to analyse the overall effect of paranoia, trial

414 by trial attributional change for each dictator, as well as order effects, and overall

415 dictator behaviour on attributions.

\section{$416 \quad 4.1$ Methodology}

417 A total of 1754 participants were included in the analysis from Study 1 and Study 2.

418 Study 3 was not preregistered. All data and analysis scripts are available online

419 (https://osf.io/u92rg/).

420 As in both previous studies, paranoia scores on the GPTS were divided into quantiles (Low, 32-36; medium, 37-44; high, 45-61; very high, 61 - 101.9) and also a group who passed GPTS scores exceeding the clinical mean (clinical, >101.9) (See

423 Figure 4).

424 Linear mixed effects models (function "Imer"; package "Ime4"; 47, ID as the random variable) were run to determine the effect of initial dictator exposure on overall $\mathrm{HI}$ and SI attributions for fair and unfair dictators. They were also used to calculate changes in $\mathrm{HI}$ and $\mathrm{SI}$ attributions for each trial relative to the first, and the overall effect of paranoia and sex on attributions. Probability distributions and uncertainty estimates of the direction of beta coefficients produced by mixed effect models were computed for $\mathrm{HI}$ and SI attributions for each trial and each dictator (using "rstanarm",

431 ID set at the random variable; 48; probability of direction fitted with "bayestestR"; 49)

432 to give a visual description of changes in $\mathrm{HI}$ and SI scores as trials continued (figure 433 5).

434 Finally, we calculated the trial where a high ( $>$ mean) attribution was made and trial

435 by trial changes to attributions when considering pre-existing paranoia (GPTS

436 score). Cumulative link models with multimodal averaging (as with Study 1 and 2)

437 were used for each dictator. Trial by trial analyses between levels of paranoia were

438 visualised separately for harmful intent and self-interest attributions for each dictator 439 (Figure 6). 


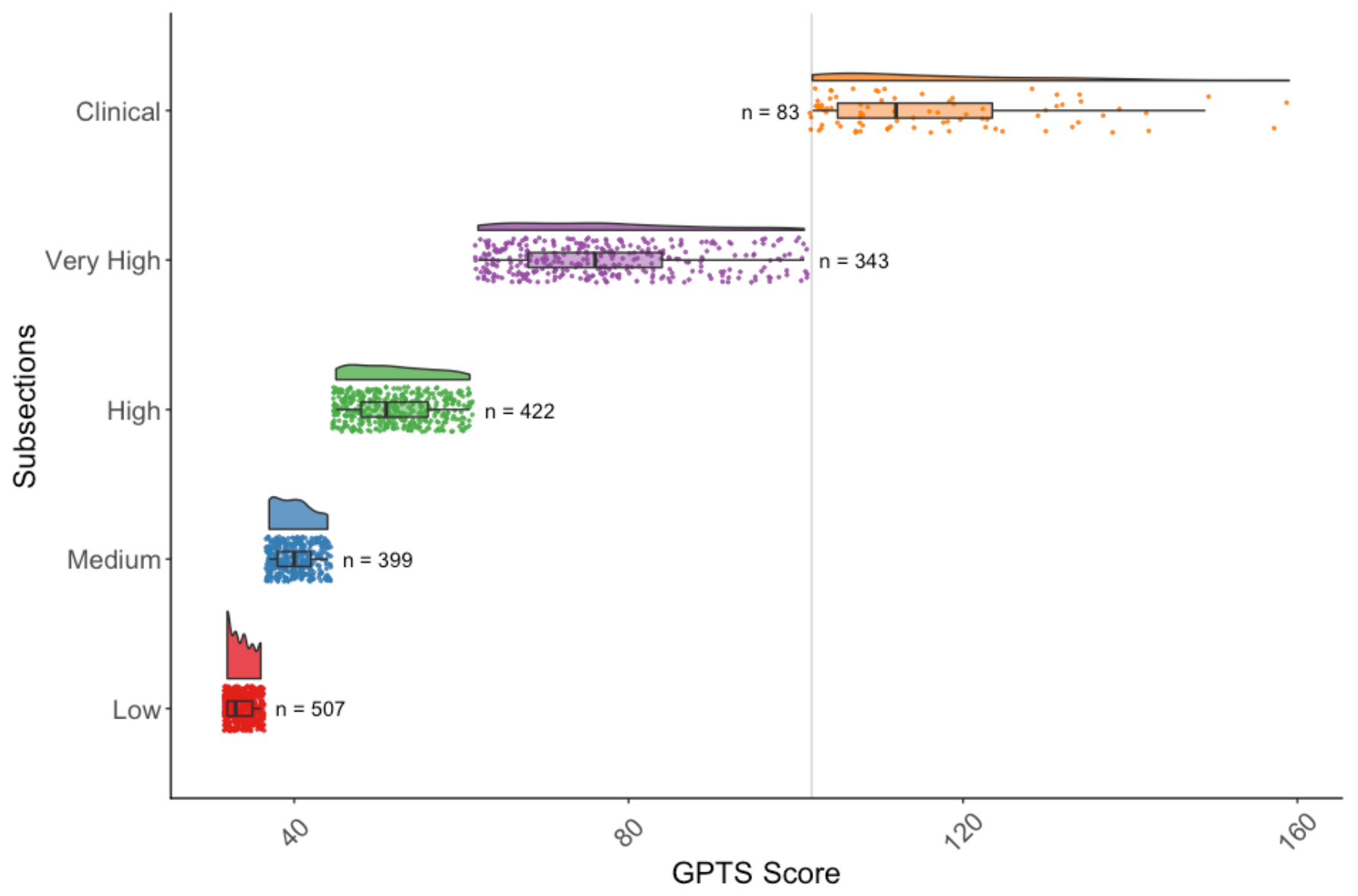

440

441 Figure 4. Rainbow cloud plot for each quartile of the Green Paranoid Thoughts

442 Scale (GPTS). The highest quantile was subdivided into those who had and hadn't

443 passed the clinical threshold (101.9) (35). The clinical division is denoted by a grey

444 line. 


\subsection{Results}

446 See Appendix D for the density distributions of scores for each dictator and trial.

\section{$447 \quad$ Order effects}

448 Being initially exposed to a more unfair dictator predicted a decrease in $\mathrm{HI}$

449 attributions for fair $(-3.61,95 \% \mathrm{Cl}:-4.38,-2.85)$ and unfair dictator conditions $(-16.70$,

$45095 \% \mathrm{Cl}:-19.50,-13.84)$ in the context of the whole population. Being initially exposed

451 to a more unfair dictator predicted a decrease in self-interest attributions when

452 playing fair $(-5.89,95 \% \mathrm{Cl}:-8.05,-3.74)$ and unfair dictator conditions $(-1.66,95 \% \mathrm{Cl}$ :

$453-2.61,-0.71)$. Paranoia predicted an increase in $\mathrm{HI}$ attributions for both dictators in

454 these models (fair dictator: $1.92,95 \% \mathrm{Cl}$ : $0.91,2.94$; unfair dictator: $3.47,95 \% \mathrm{Cl}$ :

$4551.84,5.11)$, but not SI attributions.

456 Trial by trial analysis

457 See Figure 5 (Appendix $\mathrm{E}$ for confidence intervals) for overall changes in $\mathrm{HI}$ and $\mathrm{SI}$

458 scores for each dictator from trials 1-6 across the population.

459 Paranoia predicted earlier trials in which a high $\mathrm{HI}$ score (> mean) was triggered for 460 both unfair $(-0.08,95 \% \mathrm{Cl}:-0.14,-0.01)$ and fair $(-0.08,95 \% \mathrm{Cl}:-0.14,-0.02)$ dictators.

461 This was not true for SI scores.

462 Paranoia predicted an overall decrease in scores between the first and the sixth trial

463 for fair $(-0.70,95 \% \mathrm{Cl}:-1.54,-0.03)$ but not unfair dictators. This was not true for SI

464 scores for either dictator (Figure 6 for visual summary). 


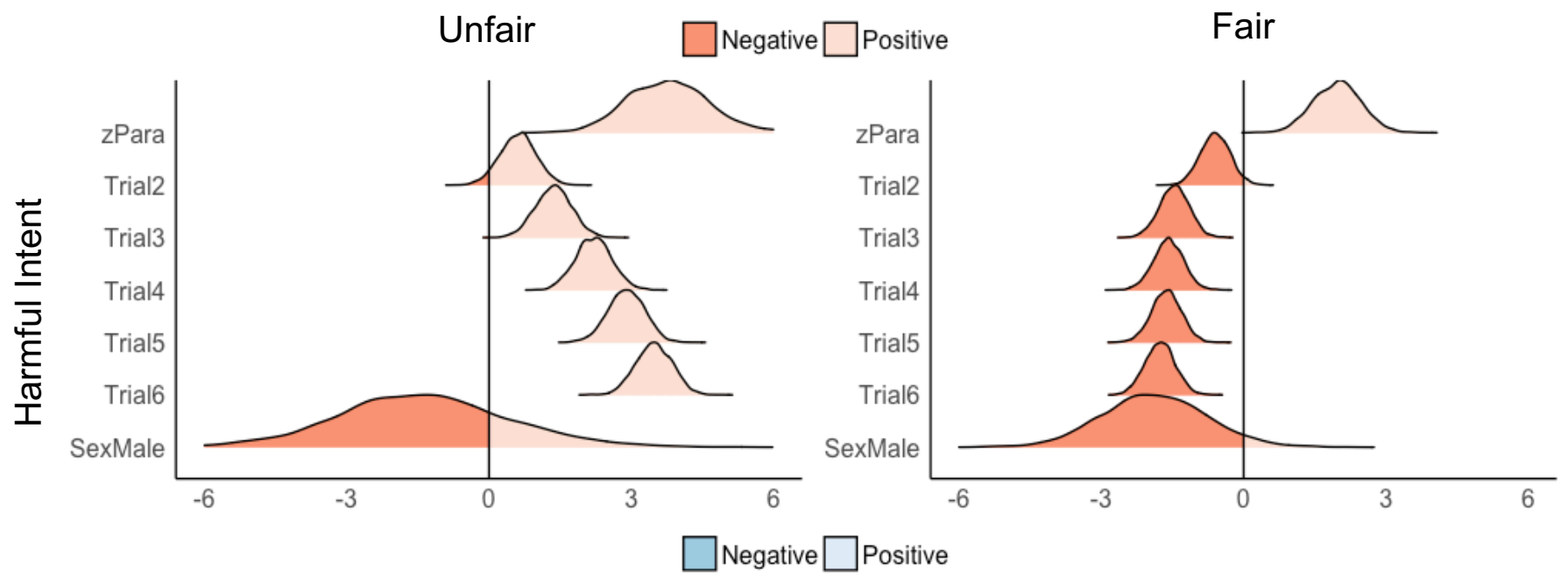

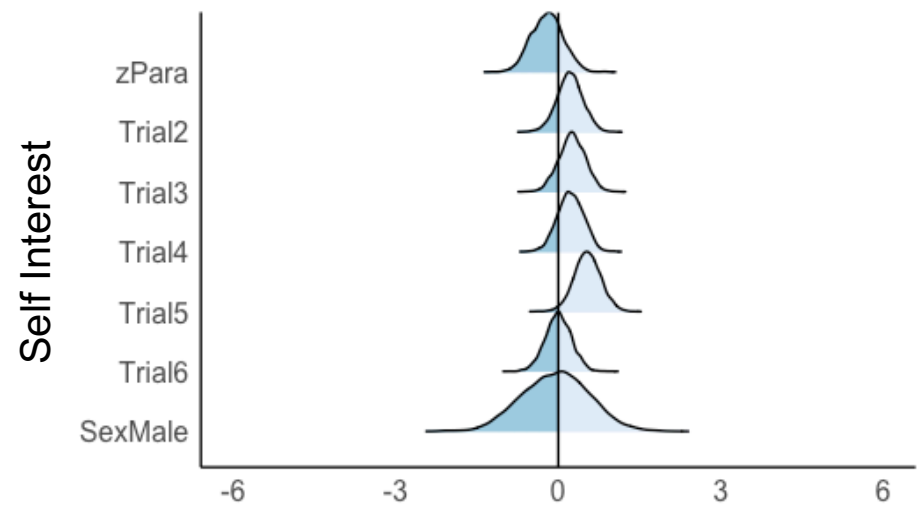

Beta estimates

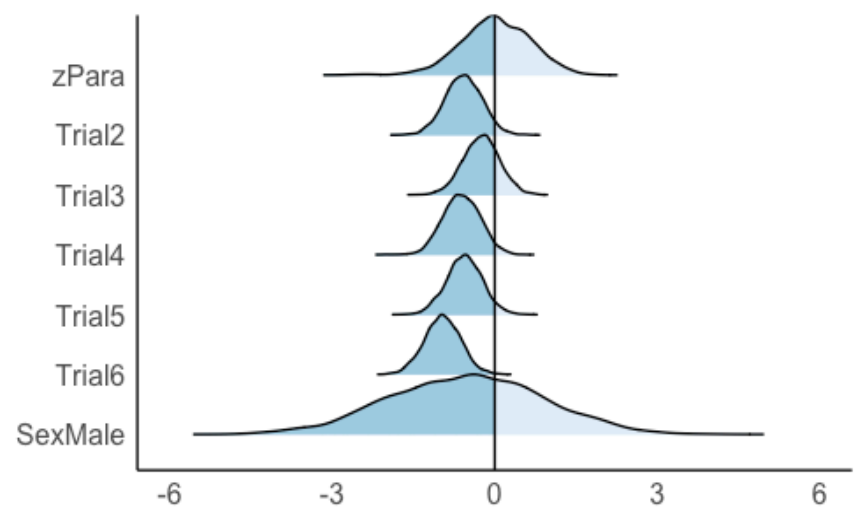

Beta estimates

467 Figure 5: Probability distributions of beta coefficient from linear mixed effects 468 models representing $\mathrm{HI}$ and $\mathrm{SI}$ attributions by unfair and fair dictators between 469 trials two to six when compared with trial one. Probability distributions of beta 470 coefficients modulated by paranoia (zPara; scaled and centred GPTS scores) and being a male (SexMale) when compared with being a female are also included. From trials three to six, unfair and fair dictators led to increases and decreases in harmful intent attributions, respectively. Social context had no impact on changes in self-

474 interest attributions over trials. 

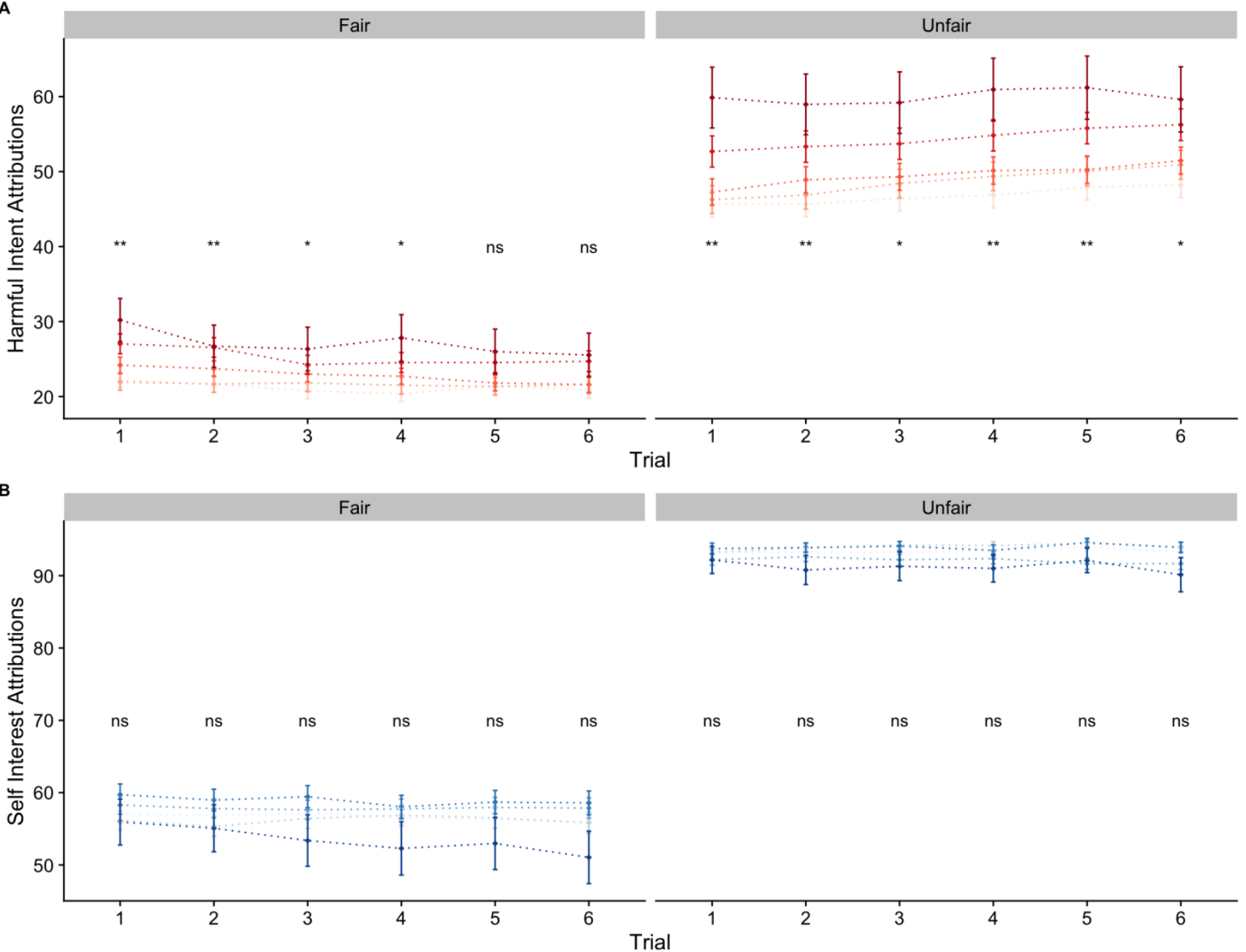

477 Figure 6: Each plot displays mean and SD for harmful intent (red) and self-

478 interest (blue) attributions, faceted by dictator. (A) Harmful Intent Attributions

479 for each trial (1-6), coloured by paranoia division. Group comparisons

480 represent HI score Paranoia for each trial. (B) Self Interest Attributions for

481 each trial (1-6), coloured by paranoia division. Group comparisons represent

482 SI score $\sim$ Paranoia for each trial. ${ }^{*}=p<0.05,{ }^{* *}=p<0.01$, ns $=$ not significant.

483 Paranoia exaggerates the magnitude of harmful intent attributions relative to social context. Higher paranoia leads to greater reductions in $\mathrm{HI}$ attributions as trials continue for fair, but not unfair dictators where initial harm $\mathrm{HI}$ attributions are sustained. This visualisation using frequentist statistics on the combined sample is confirmed by more robust information theoretic analyses from in study 3 . 


\subsection{Discussion}

493 We undertook two studies to test the sensitisation model of paranoia using a multiround Dictator game. This controlled experimental design models social inferences about the intentions of a 'dictator' (playing partner) over successive interactions and varying conditions of fair behaviours. In study one we tested the effect of selfreported paranoid beliefs on the attribution of harmful intent. In study two we tested the effect of anxiety, worry and interpersonal sensitivity in moderating these effects.

499 In line with our predictions, paranoia was associated with earlier and higher levels of

500 harmful intent attribution across all conditions, and higher levels of harmful intent attribution as partners were increasingly unfair in their division of resources. Contrary to predictions, we found no meaningful effects of anxiety or worry on the attribution of harmful intent. Moreover, trait interpersonal sensitivity was associated with a reduction in attributed harmful intent. Post-hoc analyses (study three) highlighted that paranoia was associated with greater reductions of harmful intent attributions in fair dictator conditions over six trials, but not unfair dictators. Additionally, harmful intent attributions increased over trials with unfair dictators and decreased over trials in fair dictators when analysing our population as a whole.

509 Our data provides additional evidence for the sensitisation model in paranoia. This is

510 convergent with previous game theory studies on paranoia that measured attribution

511 of harmful intent using between-subject single shot designs. In previous studies that

512 used Dictator games, paranoia predicted greater harmful intent attributions relative to 513 to partner fairness $(29,30)$. This new study replicated these findings and additionally 514 showed through the use of a within-group design and serial interactions that 515 paranoia was associated with faster and larger attributions of harmful intent relative 516 to partner fairness, suggesting increased sensitivity to perceived threat in

517 interpersonal interactions. This is in line with previous findings from studies using a 518 range of alternative paradigms. Simulated social exclusion with the 'cyberball' game 519 increased state paranoia in non-clinical individuals with high trait paranoia (50), in 520 individuals at high risk of psychosis (51), and patients with paranoid delusions (52).

521 Experience sampling studies have found that moments of subjective stress $(53,54$, 52256 ) and physiological arousal (55) predict an increase in paranoia. Similarly, 
523 immersion in a stressful social environment, either in virtual reality (56) or a genuine 524 city street (57), increased state paranoia.

525 Our data also converge with theories of social learning. Models of social impression 526 formation in healthy populations suggest that impressions of 'bad' others are more 527 volatile (changeable), and hence updated more quickly when a putatively bad agent 528 becomes fairer (58). Our findings that paranoia was associated with greater 529 reductions in harmful intent attributions in fair partner conditions provides initial 530 convergent evidence that pre-existing paranoia may amplify belief volatility.

531 Counter to our predictions, however, we did not find any effect of anxiety or worry on 532 the attribution of harmful intent. Cognitive models of paranoia (59-61) cite worry and 533 anxiety as maintaining paranoid ideation based on a range of prior evidence. Worry 534 has been found to be present at high levels in in highly paranoid people (62) and 535 psychological treatment for worry has been shown to reduce paranoia in a targeted 536 randomised controlled trial (63). Similarly, induction of stress has been shown to 537 increase state paranoia, mediated by anxiety $(6,57)$, in addition to anxiety predicting 538 higher state paranoia in ambiguous virtual environments (64). Given the strength of 539 prior evidence we think it unlikely that anxiety and worry play no part in paranoia and 540 suggest three possibilities for why no effect was found in this study. The first may be 541 that we measured harmful intent attributions for specific events and general worry 542 and anxiety may be more involved in maintaining paranoid ideation (i.e. promoting 543 paranoid rumination) than amplifying in-the-moment paranoid attributions. Secondly, 544 other predisposing factors (e.g. trauma; 17) not measured may be more relevant to 545 the relationship between general anxiety and harmful intent attributions. Finally, 546 possibility may be that the online paradigm was simply unable to capture 547 relationships between these variables. However, we find this unlikely given that we 548 detected expected relationships between variables, interactions between paranoid 549 ideation and the speed of harmful intent attributions, and found typical population 550 distributions of anxiety, worry and paranoia. Similarly, 'screen-based' studies have 551 previously reported reliable effects when testing paranoid ideation $(29-33,50-52)$.

552 Contrary to our prediction that trait interpersonal sensitivity would be associated with 553 increased harmful intent attributions, we found it was associated with reduced 554 harmful intent attributions. A recent systematic review reported a strong relationship 
between interpersonal sensitivity and trait paranoia, but a variable and unclear relationship with state paranoia (65). For example, using a general population sample, virtual reality studies have found an association between state paranoia and overall interpersonal sensitivity (66), even when adjusting for confounders $(67,68)$. However, when using 'real world' stooges, an association with state anxiety was only found with the separation anxiety subscale (69). However, we did find a positive relationship between harmful intent attributions and one subscale of the interpersonal sensitivity measure, namely separation anxiety. Insecure attachment has been found to be a robust predictor of paranoia in psychosis across multiple studies (70) potentially indicating that this finding reflected a genuine relationship, giving additional validity to our null findings from the same scale. This may suggest that the interpersonal sensitivity scale used in these studies (the Interpersonal Sensitivity Measure) may be measuring various distinct processes that are not always helpfully summarised with a single score.

We also note some limitations to this study. As with previous designs, our study used crowd-sourcing platforms. This affords us a much larger, more representative sample than university or community samples (34), with higher response rates (71), greater experimental naivety and larger chances of replication (72), although our data drew solely on a UK population. However, given our exclusion criterion (participants had to fail both questions to be removed), it is possible that some participants did not respond accurately due to poor attention, potentially leading to inflated effect sizes (34). We note however that previous studies have found online participants to produce equal or better-quality data than lab participants for the same task (73). Additionally, it is not clear to what extent those who score above the clinical mean on the paranoia scale resemble patients with paranoid delusions. Given such a large sample, it would be surprising if at least some of the high scorers didn't have delusions, although it is also the case that those most disabled by psychosis may be least able to participate in computer-based studies.

Our game theory paradigm measured harmful attributions in ambiguously motivated, loss-inducing, online interaction. One potential limitation is the extent to which participants were sceptical and believed they were being deceived by the experimenters. We found no relationship between scepticism and harmful intent 
587 attributions, and likewise our findings have replicated previous evidence using a 588 similar manipulation $(29,30)$. One additional question is the extent to which our 589 findings generalise to diverse social situations. As noted above, the results reported 590 here reflect those reported in experience sampling studies of everyday interactions 591 and immersive experimental studies, suggesting they also reflect the operation of

592 common cognitive mechanisms. However, the specific differences in how paranoia

593 manifests in online and offline contexts has yet to be tested and we feel this is 594 something that needs further research.

\subsection{Conclusion}

596 We have demonstrated that paranoid ideation leads to quicker and exaggerated 597 attributions of harmful intent, but not attributions of self-interest, in a motivationally 598 ambiguous, live online social task. Our findings support the theory of sensitisation in 599 paranoia. We provide experimental evidence that pre-existing paranoid beliefs reflect 600 a heightened sensitivity to social stress, making one more likely to attribute harmful 601 intent. We also show in a within group design that the cognitive mechanism to detect 602 social threat from unfair decisions are at least partially distinct. The finding that 603 anxiety and worry did not predict attributions of harmful intent suggests that anxiety 604 and worry may mediate paranoid rumination rather than in-the-moment attributions.

605 Additionally, while interpersonal sensitivity as a single summed score predicted 606 reduced harmful intent attributions, we did find that separation anxiety predicted 607 paranoid attributions, supporting previous work indicating a relationship between 608 insecure attachment and paranoia. We aim to test specifically clinical populations to 609 address the divide between non-clinical and clinical paranoia. At a neural level, 610 evidence of the involvement of the mesolimbic dopamine system in psychosis 611 suggest that future studies should investigate how dopamine modulates threat 612 attribution in the general as well as patient populations. 


\section{Acknowledgments}

We would like to thank Uri Hertz for kindly sending his avatar images for use in this game.

\section{Conflict of Interest Statement}

The authors declare that the research was conducted in the absence of any commercial or financial relationships that could be construed as a potential conflict of interest.

\section{Funding}

JMB is supported by the UK Medical Research Council (MR/N013700/1) and King's College London member of the MRC Doctoral Training Partnership in Biomedical Sciences.

\section{Author Contributions}

JMB initially devised the studies. JMB constructed the multi-round dictator game.

JMB and NR revised the multi-round dictator game. JMB collected the data, analysed the data and wrote initial the draft of the manuscript. JMB, QD, OR, NR, VB and MAM critically revised the manuscript. 


\subsection{References}

1. Freeman, D., \& Garety, P. A. (2000). Comments on the content of persecutory delusions: Does the definition need clarification? British Journal of Clinical Psychology. https://doi.org/10.1348/014466500163400

2. Englund, A., Morrison, P. D., Nottage, J., Hague, D., Kane, F., Bonaccorso, S., ... \& Feilding, A. (2013). Cannabidiol inhibits THC-elicited paranoid symptoms and hippocampal-dependent memory impairment. Journal of Psychopharmacology, 27(1), 19-27.

3. McKetin, R. (2018). Methamphetamine psychosis: insights from the past. Addiction, 113(8), 1522-1527.

4. Reeve, S., Emsley, R., Sheaves, B., \& Freeman, D. (2017). Disrupting sleep: the effects of sleep loss on psychotic experiences tested in an experimental study with mediation analysis. Schizophrenia bulletin, 44(3), 662-671.

5. Elliott, B., Joyce, E., \& Shorvon, S. (2009). Delusions, illusions and hallucinations in epilepsy: 2. Complex phenomena and psychosis. Epilepsy research, 85(2-3), $172-$ 186.

6. Lincoln, T. M., Peter, N., Schäfer, M., \& Moritz, S. (2009). Impact of stress on paranoia: an experimental investigation of moderators and mediators. Psychological medicine, 39(7), 1129-1139.

7. Bell, V., \& O'Driscoll, C. (2018). The network structure of paranoia in the general population. Social psychiatry and psychiatric epidemiology, 53(7), 737-744.

8. Bebbington, P. E., McBride, O., Steel, C., Kuipers, E., Radovanovič, M., Brugha, T., ... Freeman, D. (2013). The structure of paranoia in the general population. British Journal of Psychiatry, 202(6), 419-427. https://doi.org/10.1192/bjp.bp.112.119032

9. $\quad$ Freeman, D. (2007). Suspicious minds: The psychology of persecutory delusions. Clinical Psychology Review, 27(4), 425-457.

https://doi.org/10.1016/j.cpr.2006.10.004

10. Startup, H., Freeman, D., \& Garety, P. A. (2007). Persecutory delusions and catastrophic worry in psychosis: developing the understanding of delusion distress and persistence. Behaviour research and therapy, 45(3), 523-537.

11. Freeman, D., Garety, P. A., Kuipers, E., Fowler, D., Bebbington, P. E., \& Dunn, G. (2007). Acting on persecutory delusions: the importance of safety seeking. Behaviour research and therapy, 45(1), 89-99.

12. Moritz, S., Van Quaquebeke, N., \& Lincoln, T. M. (2012). Jumping to conclusions is associated with paranoia but not general suspiciousness: a comparison of two versions of the probabilistic reasoning paradigm. Schizophrenia research and treatment, 2012.

13. Bronstein, M. V., Everaert, J., Castro, A., Joormann, J., \& Cannon, T. D. (2019). Pathways to paranoia: Analytic thinking and belief flexibility. Behaviour research and therapy, 113, 18-24.

14. Freeman, D., Garety, P. A., Kuipers, E., Fowler, D., Bebbington, P. E., \& Dunn, G. (2007). Acting on persecutory delusions: the importance of safety seeking. Behaviour research and therapy, 45(1), 89-99.

15. Murphy, P., Bentall, R. P., Freeman, D., O'Rourke, S., \& Hutton, P. (2018). The paranoia as defense model of persecutory delusions: a systematic review and metaanalysis. The Lancet Psychiatry, 5(11), 913-929. 
16. Valmaggia, L. R., Freeman, D., Green, C., Garety, P., Swapp, D., Antley, A., ... \& Slater, M. (2007). Virtual reality and paranoid ideations in people with an 'at-risk mental state' for psychosis. The British Journal of Psychiatry, 191(S51), s63-s68.

17. McDonnell, J., Stahl, D., Day, F., McGuire, P., \& Valmaggia, L. R. (2018). Interpersonal sensitivity in those at clinical high risk for psychosis mediates the association between childhood bullying victimization and paranoid ideation: a virtual reality study. Schizophrenia research, 192, 89-95.

18. Collip, D., Myin-Germeys, I., \& Van Os, J. (2008). Does the concept of "sensitization" provide a plausible mechanism for the putative link between the environment and schizophrenia? Schizophrenia bulletin, 34(2), 220-225.

19. van Winkel, R., van Nierop, M., Myin-Germeys, I., \& van Os, J. (2013). Childhood trauma as a cause of psychosis: linking genes, psychology, and biology. The Canadian Journal of Psychiatry, 58(1), 44-51.

20. Kapur, S., Mizrahi, R., \& Li, M. (2005). From dopamine to salience to psychosislinking biology, pharmacology and phenomenology of psychosis. Schizophrenia research, 79(1), 59-68.

21. Howes, O. D., Bose, S. K., Turkheimer, F., Valli, I., Egerton, A., Valmaggia, L. R., ... \& McGuire, P. (2011). Dopamine synthesis capacity before onset of psychosis: a prospective [18F]-DOPA PET imaging study. American Journal of Psychiatry, 168(12), 1311-1317.

22. Howes, O. D., Kambeitz, J., Kim, E., Stahl, D., Slifstein, M., Abi-Dargham, A., \& Kapur, S. (2012). The nature of dopamine dysfunction in schizophrenia and what this means for treatment: meta-analysis of imaging studies. Archives of general psychiatry, 69(8), 776-786.

23. Howes, O. D., McCutcheon, R., Owen, M. J., \& Murray, R. M. (2017). The role of genes, stress, and dopamine in the development of schizophrenia. Biological psychiatry, 81(1), 9-20.

24. Schlier, B., Helbig-Lang, S., \& Lincoln, T. M. (2016). Anxious but thoroughly informed? No jumping-to-conclusions bias in social anxiety disorder. Cognitive Therapy and Research, 40(1), 46-56.

25. Ellett, L., Allen-Crooks, R., Stevens, A., Wildschut, T., \& Chadwick, P. (2013). A paradigm for the study of paranoia in the general population: The Prisoner's Dilemma Game. Cognition and Emotion, 27(1), 53-62.

https://doi.org/10.1080/02699931.2012.689757

26. Savulich, G., Jeanes, H., Rossides, N., Kaur, S., Zacharia, A., Robbins, T. W., \& Sahakian, B. J. (2018). Moral emotions and social economic games in paranoia. Frontiers in psychiatry, 9.

27. Haralanova, E., Haralanov, S., Beraldi, A., Möller, H. J., \& Hennig-Fast, K. (2012). Subjective emotional over-arousal to neutral social scenes in paranoid schizophrenia. European archives of psychiatry and clinical neuroscience, 262(1), 59-68.

28. Williams, L. L. M., Das, P., Liddell, B. J., Olivieri, G., Peduto, A. S., David, A. S., ... \& Harris, A. W. (2007). Fronto-limbic and autonomic disjunctions to negative emotion distinguish schizophrenia subtypes. Psychiatry Research: Neuroimaging, 155(1), 2944.

29. Raihani, N. J., \& Bell, V. (2017a). Conflict and cooperation in paranoia: a large-scale behavioral experiment. Psychological Medicine, pp. 1-11.

https://doi.org/10.1017/S0033291717003075 
30. Raihani, N. J., \& Bell, V. (2017b). Paranoia and the social representation of others: A large-scale game theory approach. Scientific Reports, 7(1), 4544.

https://doi.org/10.1038/s41598-017-04805-3

31. Greenburgh, A., Bell, V., \& Raihani, N. (2018). Paranoia and conspiracy: group cohesion increases harmful intent attribution in the Trust Game. DOI: 10.31234/osf.io/mgzjr

32. Saalfeld, V., Ramadan, Z., Bell, V., \& Raihani, N. J. (2018). Experimentally induced social threat increases paranoid thinking. Royal Society Open Science, 5(8), 180569.

33. Kahneman, D., Knetsch, J.L., Thaler, R.H. (1986) Fairness as a Constraint on Profit Seeking: Entitlements in the Market. American Economic Review. 76 (4): 728-741.

34. Berinsky, A. J., Huber, G. A., \& Lenz, G. S. (2012). Evaluating online labor markets for experimental research: Amazon. com's Mechanical Turk. Political analysis, 20(3), 351-368.

35. Green, C. E. L., Freeman, D., Kuipers, E., Bebbington, P., Fowler, D., Dunn, G., \& Garety, P. A. (2008). Measuring ideas of persecution and social reference: The Green et al. Paranoid Thought Scales (GPTS). Psychological Medicine, 38(1), 101111.

36. Statham, V., Emerson, L. M., \& Rowse, G. (2018). A Systematic Review of SelfReport Measures of Paranoia. Psychological Assessment. https://doi.org/10.1037/pas0000645

37. Grueber, C. E., Nakagawa, S., Laws, R. J., \& Jamieson, I. G. (2011). Multimodel inference in ecology and evolution: Challenges and solutions. Journal of Evolutionary Biology. John Wiley \& Sons, Ltd (10.1111). https://doi.org/10.1111/j.1420$\underline{9101.2010 .02210 . x}$

38. Burnham, K. P., \& Anderson, D. R. (2004). Multimodel inference: Understanding AIC and BIC in model selection. Sociological Methods and Research. https://doi.org/10.1177/0049124104268644

39. Galipaud, M., Gillingham, M. A. F., David, M., \& Dechaume-Moncharmont, F. X. (2014). Ecologists overestimate the importance of predictor variables in model averaging: A plea for cautious interpretations. Methods in Ecology and Evolution, 5(10), 983-991. https://doi.org/10.1111/2041-210X.12251

40. Barton, K. (2018). Package "MuMIn" Title Multi-Model Inference. Retrieved from https://cran.r-project.org/web/packages/MuMIn/MuMln.pdf

41. Team, R. D. C., \& R Development Core Team, R. (2016). R: A Language and Environment for Statistical Computing. R Foundation for Statistical Computing, 1(2.11.1), 409. https://doi.org/10.1007/978-3-540-74686-7

42. Wickham, H. (2016). ggplot2: Elegant Graphics for Data Analysis. New York: Spinger. Retrieved from https://cran.r-project.org/web/packages/g.gplot2/citation.html

43. Christensen, M. R. H. B. (2015). Package 'ordinal'. Stand, 19, 2016.

44. Boyce, P., \& Parker, G. (1989). Development of a scale to measure interpersonal sensitivity. The Australian and New Zealand Journal of Psychiatry, 23(3), 341-51.

45. Spielberger, C. D. (1989). State-Trait Anxiety Inventory: Bibliography (2nd ed.). Palo Alto, CA: Consulting Psychologists Press.

46. Meyer, T. J., Miller, M. L., Metzger, R. L., \& Borkovec, T. D. (1990). Development and validation of the penn state worry questionnaire. Behaviour research and therapy, 28(6), 487-495. 
47. Bates, D., Maechler, M., Bolker, B., Walker, S., Christensen, R. H. B., Singmann, H., ... \& Grothendieck, G. (2011). Package 'Ime4'. Linear mixed-effects models using S4 classes. $R$ package version, $1-1$.

48. Goodrich, B., Gabry, J., Ali, I., \& Brilleman, S. (2018) rstanarm: Bayesian applied regression modelling via Stan. $R$ Package Version 2.17.4.

49. Mackowski, D., Ben-Shachar, M. S., Ludecke, D. (2019) Understand and Describe Bayesian Models and Posterior Distributions using bayestest. RPackage.

50. Kesting, M. L., Bredenpohl, M., Klenke, J., Westermann, S., \& Lincoln, T. M. (2013). The impact of social stress on self-esteem and paranoid ideation. Journal of behavior therapy and experimental psychiatry, 44(1), 122-128.

51. Lincoln, T. M., Sundag, J., Schlier, B., \& Karow, A. (2017). The relevance of emotion regulation in explaining why social exclusion triggers paranoia in individuals at clinical high risk of psychosis. Schizophrenia bulletin, 44(4), 757-767.

52. Sundag, J., Ascone, L., \& Lincoln, T. M. (2018). The predictive value of early maladaptive schemas in paranoid responses to social stress. Clinical psychology \& psychotherapy, 25(1), 65-75.

53. Kramer, I., Simons, C. J., Wigman, J. T., Collip, D., Jacobs, N., Derom, C., ... \& Wichers, M. (2013). Time-lagged moment-to-moment interplay between negative affect and paranoia: new insights in the affective pathway to psychosis. Schizophrenia bulletin, 40(2), 278-286.

54. Barrantes-Vidal, N., Chun, C. A., Myin-Germeys, I., \& Kwapil, T. R. (2013). Psychometric schizotypy predicts psychotic-like, paranoid, and negative symptoms in daily life. Journal of Abnormal Psychology, 122(4), 1077.

55. Schlier, B., Krkovic, K., Clamor, A., \& Lincoln, T. M. (2019). Autonomic arousal during psychosis spectrum experiences: Results from a high-resolution ambulatory assessment study over the course of symptom on-and offset. Schizophrenia Research.

56. Veling, W., Pot-Kolder, R., Counotte, J., van Os, J., \& van der Gaag, M. (2016). Environmental social stress, paranoia and psychosis liability: a virtual reality study. Schizophrenia bulletin, 42(6), 1363-1371.

57. Ellett, L., Freeman, D., \& Garety, P. A. (2008). The psychological effect of an urban environment on individuals with persecutory delusions: the Camberwell walk study. Schizophrenia research, 99(1-3), 77-84.

58. Siegel, J. Z., Mathys, C., Rutledge, R. B., \& Crockett, M. J. (2018). Beliefs about bad people are volatile. Nature Human Behaviour, 2(10), 750.

59. Freeman, D., Stahl, D., McManus, S., Meltzer, H., Brugha, T., Wiles, N., \& Bebbington, P. (2012). Insomnia, worry, anxiety and depression as predictors of the occurrence and persistence of paranoid thinking. Social psychiatry and psychiatric epidemiology, 47(8), 1195-1203.

60. Freeman, D. (2016). Persecutory delusions: a cognitive perspective on understanding and treatment. The Lancet Psychiatry, 3(7), 685-692.

61. Sun, X., So, S. H. W., Chiu, C. D., Chan, R. C. K., \& Leung, P. W. L. (2018). Paranoia and anxiety: A cluster analysis in a non-clinical sample and the relationship with worry processes. Schizophrenia research, 197, 144-149.

62. Startup, H., Pugh, K., Dunn, G., Cordwell, J., Mander, H., Černis, E., ... \& Freeman, D. (2016). Worry processes in patients with persecutory delusions. British Journal of Clinical Psychology, 55(4), 387-400. 
63. Freeman, D., Dunn, G., Startup, H., Pugh, K., Cordwell, J., Mander, H., ... \& Kingdon, D. (2015). Effects of cognitive behaviour therapy for worry on persecutory delusions in patients with psychosis (WIT): a parallel, single-blind, randomised controlled trial with a mediation analysis. The Lancet Psychiatry, 2(4), 305-313.

64. Freeman, D, Slater, M, Bebbington, PE, Garety, PA, Kuipers, E, Fowler, D et al. (2003) Can virtual reality be used to investigate persecutory ideation? The Journal of Nervous and Mental Disease 191(8), 509-514.

65. Meisel, S. F., Garety, P. A., Stahl, D., \& Valmaggia, L. R. (2018). Interpersonal processes in paranoia: a systematic review. Psychological medicine, 48(14), 22992312.

66. Freeman, D., Pugh, K., Vorontsova, N., Antley, A., \& Slater, M. (2010). Testing the continuum of delusional beliefs: An experimental study using virtual reality. Journal of abnormal psychology, 119(1), 83.

67. Freeman, D, Gittins, M, Pugh, K, Antley, A, Slater, M and Dunn, G (2008) What makes one-person paranoid and another person anxious? The differential prediction of social anxiety and persecutory ideation in an experimental situation. Psychological Medicine 38(8), 1121-1132.

68. Freeman, D, Pugh, K, Antley, A, Slater, M, Bebbington, P, Gittins, M et al. (2008) Virtual reality study of paranoid thinking in the general population. The British Journal of Psychiatry 192(4), 258-263.

69. Green, CE, Freeman, D, Kuipers, E, Bebbington, P, Fowler, D, Dunn, G et al. (2011) Paranoid explanations of experience: a novel experimental study. Behavioural and Cognitive Psychotherapy 39(1), 21.

70. Lavin, R., Bucci, S., Varese, F., \& Berry, K. (2019). The relationship between insecure attachment and paranoia in psychosis: A systematic literature review. British Journal of Clinical Psychology.

71. Peer, E., Brandimarte, L., Samat, S., \& Acquisti, A. (2017). Beyond the Turk: Alternative platforms for crowdsourcing behavioral research. Journal of Experimental Social Psychology, 70, 153-163.

72. Crump, M. J., McDonnell, J. V., \& Gureckis, T. M. (2013). Evaluating Amazon's Mechanical Turk as a tool for experimental behavioral research. PloS one, 8(3), e57410.

73. Hauser, D. J., \& Schwarz, N. (2016). Attentive Turkers: MTurk participants perform better on online attention checks than do subject pool participants. Behavior research methods, 48(1), 400-407. 


\subsection{Supplementary Material}

7.1 Appendix A - The multi-round dictator game task schematic for one partner.

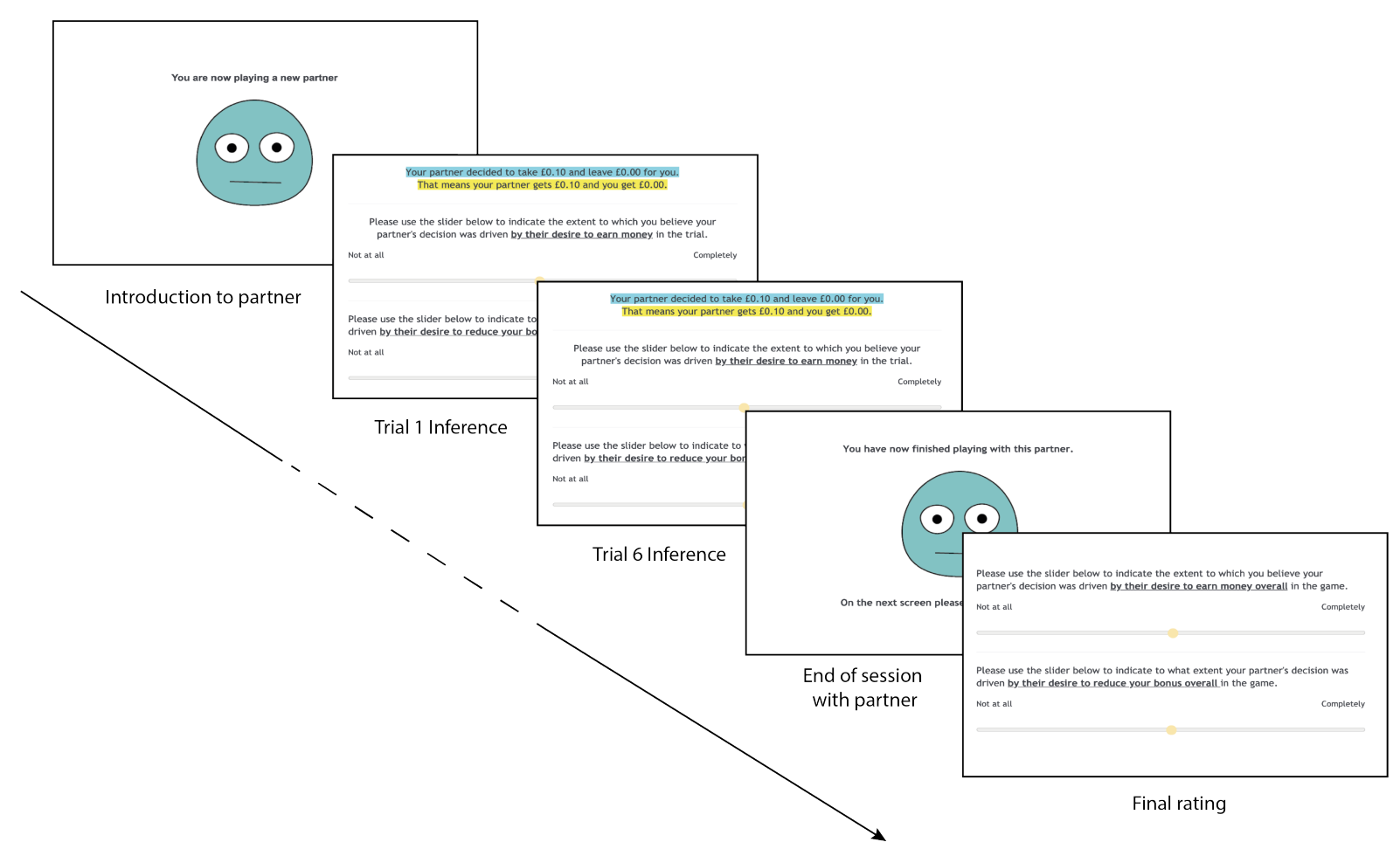


7.2 Appendix B - Trials-to-peak-decision for Harmful intent and Self-Interest scores

Variables affecting earlier trial-to-peak-decision for Harmful Intent attributions within unfair and fair dictator decisions in the multi-round dictator game

(Study 1). Trials where 53.51 (unfair) or 24.26 (fair) was first triggered were coded like so: $6<5<4<3<2<1<0$, where 0 means over the mean was never scored or an earlier trial scored over the mean. Relative Importance is the probability that the term in question is a component of the true best model.

\begin{tabular}{|c|c|c|c|c|c|}
\hline \multirow[t]{2}{*}{ Parameter } & \multirow[t]{2}{*}{ Estimate } & \multirow{2}{*}{$\begin{array}{c}\text { Standard } \\
\text { Error }\end{array}$} & \multicolumn{2}{|c|}{$95 \% \mathrm{Cl}$} & \multirow{2}{*}{$\begin{array}{l}\text { Relative } \\
\text { Importance }\end{array}$} \\
\hline & & & Lower & Upper & \\
\hline \multicolumn{6}{|l|}{ Unfair Dictator } \\
\hline Intercept 6|5 & -2.49 & 0.09 & -2.66 & -2.32 & \\
\hline Intercept 5|4 & -2.40 & 0.08 & -2.57 & -2.24 & \\
\hline Intercept $4 \mid 3$ & -2.37 & 0.08 & -2.54 & -2.21 & \\
\hline Intercept 3|2 & -2.33 & 0.08 & -2.50 & -2.17 & \\
\hline Intercept 2|1 & -2.30 & 0.08 & -2.47 & -2.14 & \\
\hline Intercept $1 \mid 0$ & -2.28 & 0.08 & -2.44 & -2.11 & \\
\hline Paranoia (Z score) & -0.12 & 0.05 & -0.21 & -0.02 & 1 \\
\hline Age & -0.01 & 0.02 & -0.06 & 0.02 & 0.32 \\
\hline Sex (Male | Female) & 0.01 & 0.05 & -0.14 & 0.26 & 0.21 \\
\hline \multicolumn{6}{|l|}{ Fair Dictator } \\
\hline Intercept 6|5 & -2.61 & 0.10 & -2.81 & -2.40 & \\
\hline Intercept 5|4 & -2.51 & 0.10 & -2.71 & -2.31 & \\
\hline Intercept 4|3 & -2.47 & 0.10 & -2.66 & -2.27 & \\
\hline Intercept $3 \mid 2$ & -2.43 & 0.10 & -2.63 & -2.23 & \\
\hline Intercept 2|1 & -2.39 & 0.10 & -2.59 & -2.20 & \\
\hline Intercept 1|0 & -2.38 & 0.10 & -2.58 & -2.18 & \\
\hline Age & -0.02 & 0.02 & -0.07 & 0.01 & 0.69 \\
\hline Paranoia (Z score) & -0.06 & 0.06 & -0.19 & 0.01 & 0.55 \\
\hline Sex (Male | Female) & 0.01 & 0.04 & -0.15 & 0.26 & 0.13 \\
\hline
\end{tabular}


Variables effecting earlier trial-to-peak-decision for Self-Interest within unfair dictator decisions in a multi-round dictator game (Study 1). Trials where 60 was triggered were coded like so: $6<5<4<3<2<1<0$, where 0 means 60 was never scored or an earlier trial scored 60 . Relative Importance is the probability that the term in question is a component of the true best model.

\begin{tabular}{|l|c|c|c|c|c|}
\hline Parameter & \multirow{2}{*}{ Estimate } & \multirow{2}{*}{ Standard } & \multicolumn{2}{|c|}{$95 \% \mathrm{Cl}$} & Relative \\
\cline { 4 - 5 } & & & Eower & Upper & Importance \\
\hline Intercept 6|5 & -1.64 & 0.04 & -1.72 & -1.57 & \\
\hline Intercept 5|4 & -1.63 & 0.04 & -1.70 & -1.55 & \\
\hline Intercept 4|3 & -1.63 & 0.04 & -1.70 & -1.55 & \\
\hline Intercept 3|0 & -1.63 & 0.04 & -1.70 & -1.55 & \\
\hline Paranoia (Z score) & 0.01 & 0.08 & -0.14 & 0.17 & 0.21 \\
\hline Age & 0.01 & 0.08 & -0.15 & 0.16 & 0.21 \\
\hline
\end{tabular}

Variables effecting earlier trial-to-peak-decision for Self-Interest within fair dictator decisions in a multi-round dictator game (Study 1). Trials where 60 was triggered were coded like so: $6<5<4<3<2<1<0$, where 0 means 60 was never scored or an earlier trial scored 60 . Relative Importance is the probability that the term in question is a component of the true best model.

\begin{tabular}{|c|c|c|c|c|c|}
\hline \multirow[t]{2}{*}{ Parameter } & \multirow[t]{2}{*}{ Estimate } & \multirow{2}{*}{$\begin{array}{c}\text { Standard } \\
\text { Error }\end{array}$} & \multicolumn{2}{|c|}{$95 \% \mathrm{Cl}$} & \multirow{2}{*}{$\begin{array}{c}\text { Relative } \\
\text { Importance }\end{array}$} \\
\hline & & & Lower & Upper & \\
\hline Intercept $6 \mid 5$ & -2.37 & 0.05 & -2.47 & -2.26 & \\
\hline Intercept $5 \mid 4$ & -2.27 & 0.05 & -2.38 & -2.17 & \\
\hline Intercept $4 \mid 3$ & -2.23 & 0.05 & -2.33 & -2.13 & \\
\hline Intercept $3 \mid 2$ & -2.20 & 0.05 & -2.30 & -2.10 & \\
\hline Intercept 2|1 & -2.18 & 0.05 & -2.28 & -2.08 & \\
\hline Intercept 1/0 & -2.17 & 0.05 & -2.26 & -2.07 & \\
\hline Age & 0.04 & 0.10 & -0.15 & 0.23 & 0.22 \\
\hline Sex (Male | Female) & 0.03 & 0.09 & -0.16 & 0.22 & 0.22 \\
\hline
\end{tabular}


7.2 Appendix C - Correlation coefficients of all baseline variables. Top panel: Age, GPTS, STAI-T, IPS (total measure), and PSWQ. Bottom panel: GPTS, Interpersonal awareness, Need for Attachment, Separation Anxiety, Timidity, and Fragile Inner Self Subscale of the IPS.
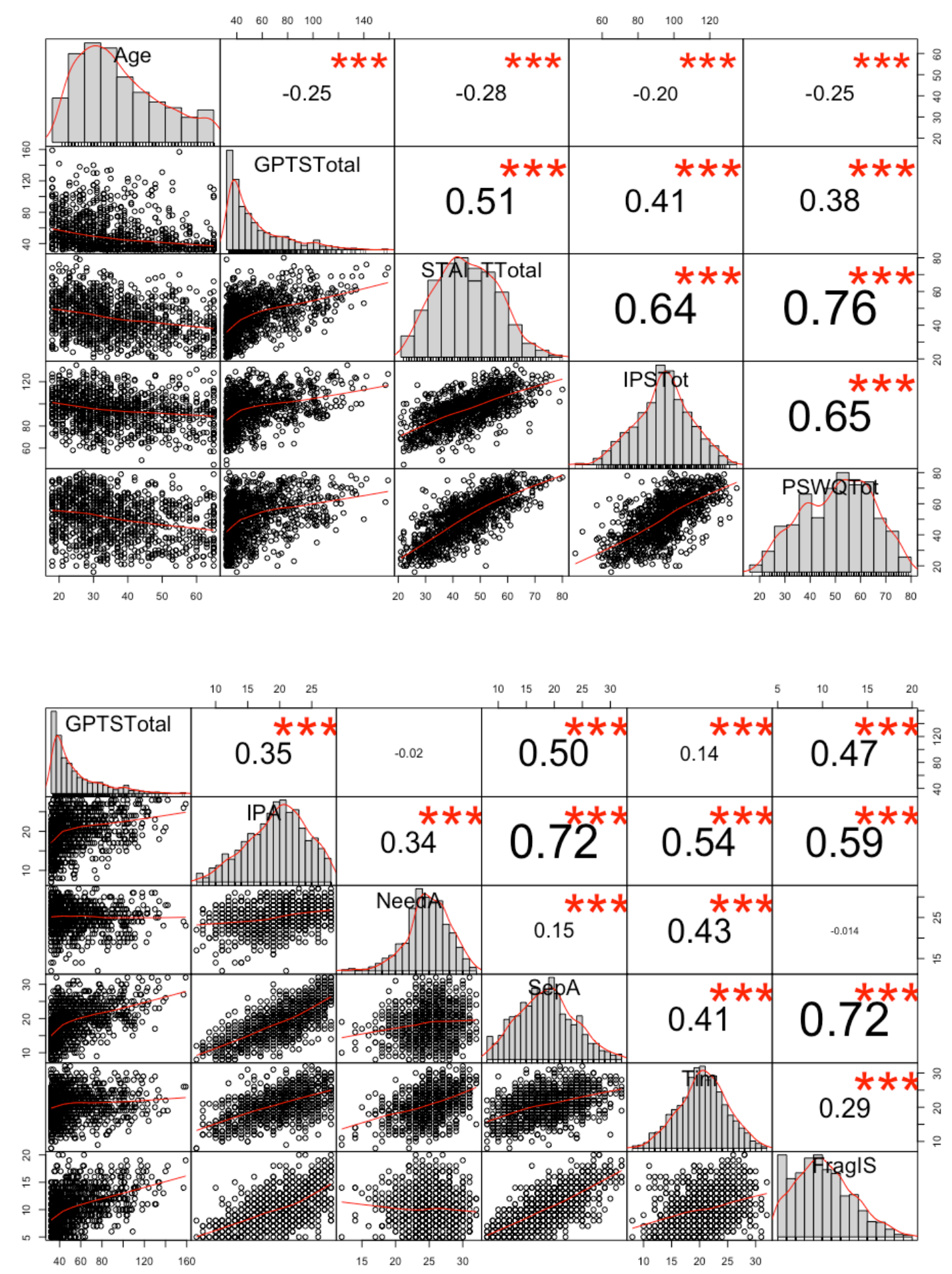
7.3 Appendix D - A \& B: Density distributions for Harmful Intent scores within each trial (1-6) for unfair $(A)$ and fair $(B)$ dictators for each level of paranoia. C \& D:

Density distributions for Self Interest scores within each trial (1-6) for unfair (C) and fair (D) dictators for each level of paranoia.

A

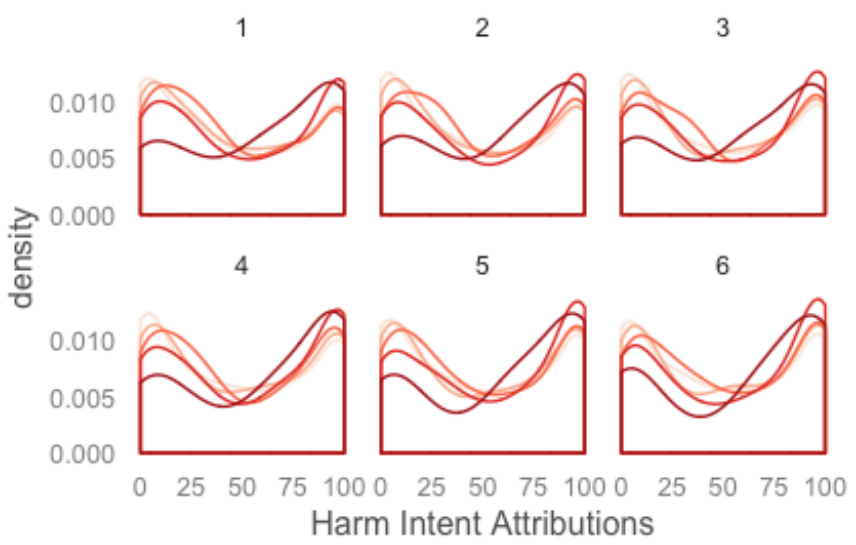

C

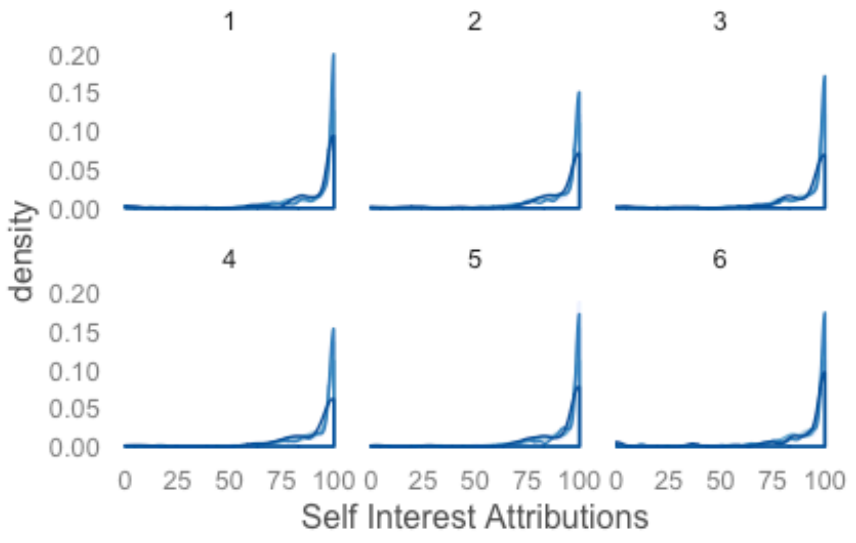

B

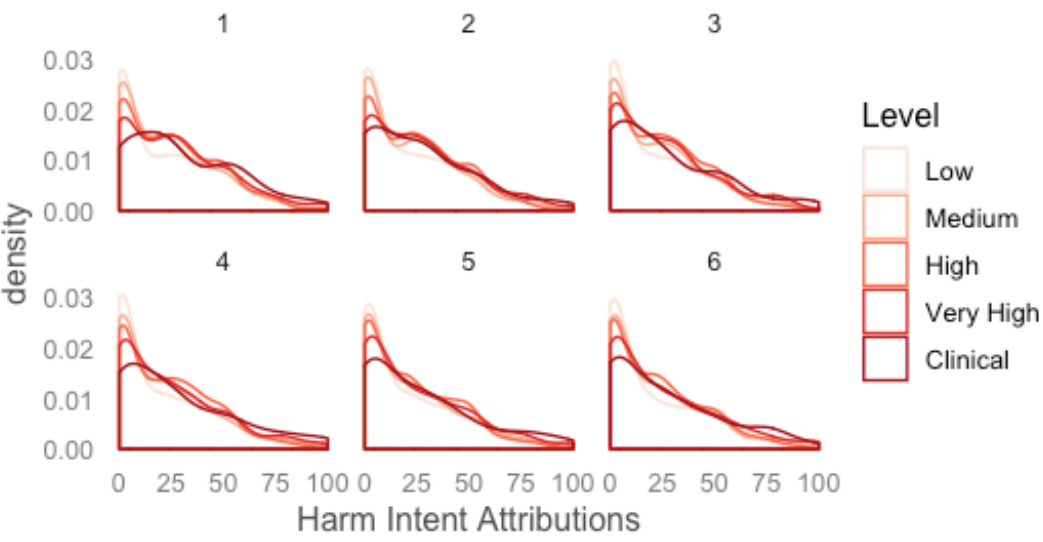

D

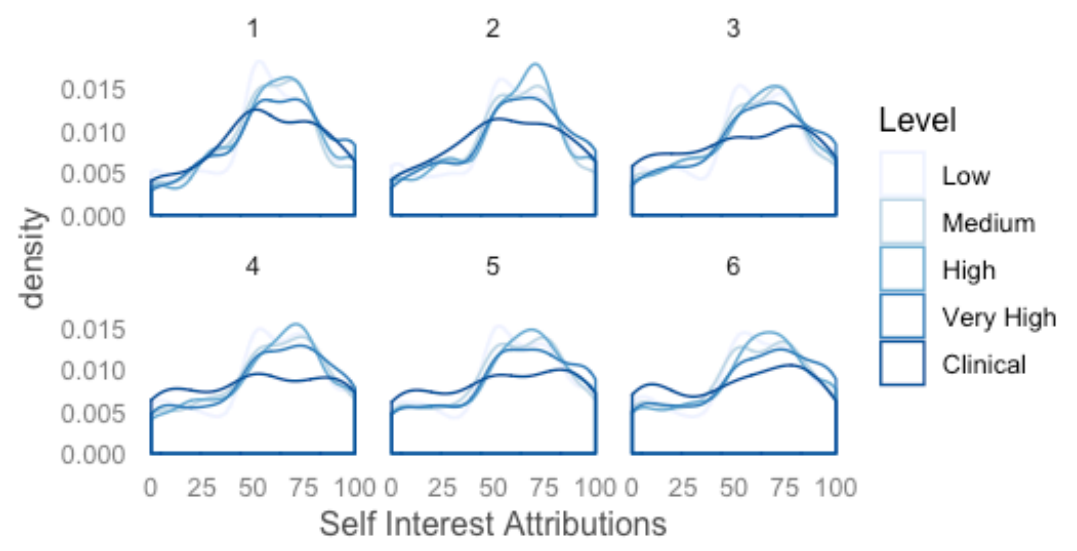


7.4 Appendix E - Beta coefficient 95\% confidence intervals from linear mixed effects models for harmful intent and self-interest attributions for paranoia, dictator, and sex.

\begin{tabular}{|l|l|l|l|l|l|l|l|l|}
\hline & \multicolumn{4}{|c|}{ Harmful Intent Attributions } & \multicolumn{3}{c|}{ Self Interest Attributions } \\
\hline & Unfair Dictator & \multicolumn{1}{|l|}{ Fair Dictator } & \multicolumn{2}{l|}{ Unfair Dictator } & \multicolumn{2}{|c|}{ Fair Dictator } \\
\hline $95 \% \mathrm{Cl}$ & $2.50 \%$ & $97.50 \%$ & $2.50 \%$ & $97.50 \%$ & $2.50 \%$ & $97.50 \%$ & $2.50 \%$ & $97.50 \%$ \\
\hline. sig01 & 34.85 & 37.27 & 20.73 & 22.19 & 11.03 & 11.83 & 25.76 & 27.57 \\
\hline sigma & 11.09 & 11.42 & 9.04 & 9.31 & 6.93 & 7.14 & 9.52 & 9.81 \\
\hline (Intercept) & 46.66 & 51.04 & 23.31 & 25.99 & 92.29 & 93.80 & 56.23 & 59.51 \\
\hline zPara & 1.93 & 5.34 & 0.91 & 2.96 & -0.74 & 0.36 & -1.29 & 1.24 \\
\hline Trial2 & -0.13 & 1.36 & -1.21 & 0.00 & -0.25 & 0.68 & -1.21 & 0.06 \\
\hline Trial3 & 0.62 & 2.11 & -2.07 & -0.86 & -0.21 & 0.72 & -0.89 & 0.39 \\
\hline Trial4 & 1.46 & 2.95 & -2.19 & -0.98 & -0.23 & 0.70 & -1.25 & 0.03 \\
\hline Trial5 & 2.16 & 3.65 & -2.23 & -1.02 & 0.08 & 1.01 & -1.20 & 0.08 \\
\hline Trial6 & 2.75 & 4.24 & -2.38 & -1.16 & -0.46 & 0.47 & -1.60 & -0.33 \\
\hline SexMale & -5.22 & 1.85 & -4.03 & 0.20 & -1.23 & 1.06 & -3.29 & 1.95 \\
\hline
\end{tabular}

